\title{
Stochastic PDEs and lack of regularity
}

A surface growth equation with noise: existence, uniqueness, and blow-up

\author{
Dirk Blömker · Marco Romito
}

Received: date / Accepted: date

\begin{abstract}
We review results on the existence and uniqueness for a surface growth model with or without space-time white noise. If the surface is a graph, then this model has striking similarities to the three dimensional Navier-Stokes equations in terms of energy estimates and scaling properties, and in both models the question of uniqueness of global weak solutions remains open.

In the physically relevant dimension $d=2$ and with the physically relevant space-time white noise driving the equation, the direct fixed-point argument for mild solutions fails, as there is not sufficient regularity for the stochastic forcing. The situation is the simplest case where the method of regularity structures introduced by Martin Hairer can be applied, although we follow here a significantly simpler approach to highlight the key problems. Using spectral Galerkin method or any other type of regularization of the noise, one can give a rigorous meaning to the stochastic PDE and show existence and uniqueness of local solutions in that setting. Moreover, several types of regularization seem to yield all the same solution.

We finally comment briefly on possible blow up phenomena and show with a simple argument that many complex-valued solutions actually do blow up in finite time. This shows that energy estimates alone are not enough to verify global uniqueness of solutions. Results in this direction are known already for the 3D-Navier Stokes by Li and Sinai, treating complex valued solutions, and more recently by Tao by constructing an equation of NavierStokes type with blow up.
\end{abstract}

Keywords Local existence and uniqueness $\cdot$ surface growth model $\cdot$ regularization of noise $\cdot$ fixed point argument $\cdot$ mild solution

Mathematics Subject Classification (2010) 35B33 - 35B45 - 35B65 - 35K55 - 35Qxx * $35 \mathrm{R} 60 \cdot 60 \mathrm{G} 15 \cdot 60 \mathrm{H} 15$

D. Blömker

Institut für Mathematik, Universität Augsburg, D-86135 Augsburg, Germany

E-mail: dirk.bloemker@math.uni-augsburg.de

M. Romito

Dipartimento di Matematica, Università di Pisa, Largo Bruno Pontecorvo 5, I-56127 Pisa, Italia

E-mail: romito@dm.unipi.it 


\section{Contents}

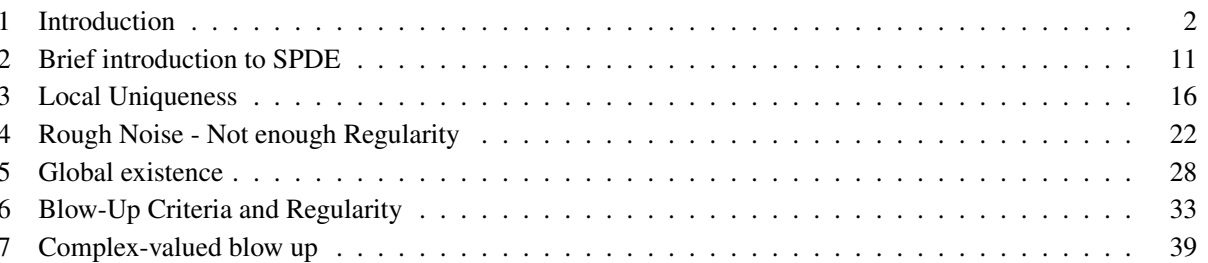

\section{Introduction}

The analysis of mathematical models for the study of surface growth phenomena has attracted attention in recent years from both the physical and the mathematical communities. In this paper we focus on one special 4-th order equations with quadratic nonlinearity that does not allow for maximum principle or gradient flow structures and has striking similarities to the Navier-Stokes equation in dimension 3 and the Kadar-Parisi-Zhang (KPZ) equation.

The equation is of the type

$$
\partial_{t} h=-\Delta^{2} h-\Delta|\nabla h|^{2}+\xi
$$

where $h(t, \cdot)$ is the graph of the surface at time $t>0$ over a $d$-dimensional domain (with $d=1,2$ ), and $\xi$ is space-time white noise. Here $\Delta$ denotes the Laplacian and $\nabla$ the gradient of $h$.

In Section 2 we give a brief introduction to stochastic partial differential equations (SPDEs) and space-time white noise. For the whole paper we focus on the relatively simple setting of additive noise only, which is present in most of the applications.

\subsection{Physical Model}

Let us first discuss physical properties of the model. For mathematical details on the model see the exposition in Section 1.3. For additional references see [88, 23, 81, 80,46, 89, 87, 104] or Da Prato \& Zabczyk [32, Sec. 13.14]. For general surveys on surface growth processes and molecular beam epitaxy see the classical references by Barabási \& Stanley [3] or HalpinHealy \& Zhang [56].

Equation (1), which is sometimes referred to as a mass-conservative version of the KPZ model, arises in several models for surface growth. The two-dimensional version was for example suggested in $[87,89,88]$ as a phenomenological model for the growth of an amorphous surface $\left(\mathrm{Zr}_{65} \mathrm{Al}_{7,5} \mathrm{Cu}_{27,5}\right)$. But similar equations appear also in the formation of sand or snow ripples. Later the equation has also become a model for ion-sputtering, where a surface is eroded by a ion-beam, see Cuerno \& Barabási [27], Castro et al. [23] or [81,80]. The one-dimensional equation appeared also as a model for the boundaries of terraces in the epitaxy of Silicon [46]. Further details on the model can be found in $[68,110,117,104]$. 
Derivation of the model. In the literature there are two possible methods suggested for the derivation of our model. The first one is a symmetry argument. One assumes that on $\mathbb{R}^{d}$ the equation only contains terms that are invariant under all symmetry operations, which do not change the growth process. These are for example rotations of the underlying domain, reflections $x_{i} \mapsto-x_{i}$, or translations in both $x$ and $h$. Furthermore, the equation should conserve mass, as the model is supposed to be in a moving frame following the average growth rate of the surface. So the right-hand side needs to be a divergence.

There are only two linear and two quadratic terms that satisfy all these requirements. Higher order terms are usually neglected in the physics literature, for example by considering a small gradient expansion of the nonlinearity. Two terms are already present in our equation, which are $-\Delta^{2} h$ and $-\Delta|\nabla h|^{2}$. The missing linear term $-\Delta u$ is of lower order and usually associated with the formation of hills. The other quadratic term is the determinant of the Hessian of $h$, which has a completely different nature than the term studied here. We comment on both terms in more detail in Section 1.3.

Another approach that leads to models of these type is more geometric in nature. First consider motion on the surface by surface diffusion, which leads in a linearisation to the $-\Delta^{2}$. The nonlinear term then arises by the more complicated geometric argument that particles are attracted to the surface and not fall down vertically. This leads to an effective uphill current of mass, depending on the slope of the surface, which in first order gives $-\Delta u$ and in second order the non-linearity considered here.

Surface Roughness. The typical physical quantity of interest is the roughness of the surface. This is measured as the averaged quadratic deviation from the spatial mean of the surface. As one considers a moving frame, this is usually just the expectation of the squared $L^{2}$-norm.

The question is now to determine characteristic exponents $\alpha, \beta$, and $z$, also determining the so called universality class of the model, such that for a system size of order $L$

$$
\text { mean surface roughness } \sim\left\{\begin{array}{l}
t^{\beta}: t \ll L^{z} \\
L^{\alpha}: t \gg L^{z}
\end{array}\right.
$$

Unfortunately our mathematical analysis of the problem is at an earlier stage and does not allow yet to give rigorous conclusions on these questions. For analytic estimates see for example [14], or we refer the reader to the rich physics literature.

Hill formation and coarsening. Characteristic of all these models with an additional lower order linearity is the formation of parabola shaped hills, with sharp, almost corner like, valleys in between. See Figure 1 for a one-dimensional numerical calculation. But also in several two dimensional experiments, due to an initial linear instability of the flat surface, the solution exhibits many paraboloid hills with sharp valleys in between. Over time these hills tend to coarsen, when larger hills just bury the smaller ones. See [84] for a reduced model in one dimension given by the position of the interfaces. In none of the numerical or real world experiments chaotic or irregular behaviour was observed, which is on the contrast to many other well-known and quite similar models of surface growth like Kuramoto-Sivashinsky, which models the propagation of flame fronts. There the surface usually exhibits quite complicated dynamics. 
1.2 Obstacles and difficulties in the mathematical analysis of the model

A crucial mathematical open problem for our model equation, with or without random perturbations, is the uniqueness of global solutions. Indeed, in one space dimension it is possible to show existence of locally unique and smooth solutions, as well as of global weak solutions, but unfortunately there is a gap in the regularity requirements for the initial condition for the two types of solutions. As such the problem is reminiscent of the celebrated millennium problem of existence of global smooth solutions for the Navier-Stokes equations. Here in two spatial dimensions the problem is even harder, at least at the level of existence of weak solutions, due to some $a$-priori estimates that fail to be true in dimension higher than one.

In two dimension, for the stochastic PDE, there is an additional difficulty: the driving force, which is space-time white noise, is a rough forcing and in principle, even in a weak formulation, the solution does not acquire enough regularity to give a meaning to the nonlinearity. In the following, we shall discuss all these points in more detail.

Local solutions. Local existence and uniqueness of solutions can be straightforwardly proved by a fixed point theorem, both with and without noise perturbations. A preliminary result on local uniqueness of $W^{1,4}$-valued solutions for the stochastic PDE in dimension $d=1,2$ can be found in [10]. The existence and uniqueness of a regularized problem with a cut-off in the nonlinearity in dimension $d=2$ has been studied in Hoppe, Linz \& Litvinov [58]. In [16] in the one dimensional case the uniqueness of local solutions was established with initial values in the critical Hilbert space $H^{1 / 2}$ (see Section 3.1 for more details on the scaling invariance of the equation and quantities critical for the scaling, namely quantities invariant by scaling).

Another approach for local uniqueness and global existence based on Galerkin approximations combined with local monotonicity and generalized coercivity conditions is established by the application of an abstract result by Röckner et al. [74,91]. But this is, at least in terms of initial conditions, not yet optimal, as it needs initial conditions with two spatial derivatives, i.e. in $H^{2}$.

Existence and uniqueness in the largest possible critical space can be achieved using the ideas introduced by Koch \& Tataru [65] for the Navier-Stokes equation. It has been implemented for the surface growth equation in [17], and we summarize it in Section 3. The result yields that the largest critical space of initial conditions where local existence and uniqueness holds, is equivalent to the homogeneous Besov space $\dot{B}_{\infty}^{0, \infty}$. This space contains for example the critical spaces $C^{0}$ of continuous functions as well as the Hilbert-space $H^{d / 2}$ with $d / 2$ fractional derivatives that are still square integrable. But, as we will see, there still remains a wide gap of regularity from the largest critical space to the global a-priori bounds in $L^{2}$ for $d=1$ and $H^{-1}$ for $d=2$ that are used to show global existence of weak solutions.

Weak solutions. For $d=1$ existence of global weak solutions follows by an $a$-priori estimate of the the mean squared height of the surface (see Section 1.5) and by standard compactness methods. For the stochastic PDE this can be extended to compactness in spaces of probability measures. The existence of global weak solutions on bounded domains has been first studied for our equation in [12] (see also the references therein), based on spectral Galerkin methods. See also the discussion of the improved results in [9] below.

Unfortunately the estimate fails to hold in larger spatial dimensions. Without noise, Stein \& Winkler [108] used Rothe's method, which is a fully implicit discretization of the timederivative, in order to verify the existence of a global weak solution that is only uniformly 
bounded in a weak topology given by the $H^{-1}$-norm. This result has been extended by Winkler [120] to the two-dimensional case, using energy type estimates for the Lyapunov functional $\int \mathrm{e}^{h} d x$ (cf. Section 1.6). Existence of weak solutions of the stochastic PDE in the physical dimension two is, so far, an open problem, which should be solvable by combining the stochastic approach with the results of $[108,120]$.

Convergence to equilibrium. Existence of weak solutions for the stochastic PDE has been significantly improved in [9] using the method developed by Flandoli \& Romito [42,44] (see also the related results $[43,92,94,96]$ ) in order to verify the existence of a solution that defines a Markov process. We review this in Section 5. For related results see $[30,35]$ and [49]. The method allows to define a Markov evolution associated to the equation and thus to introduce invariant measures. These are random initial distributions that are invariant under the dynamics. It turns out that for any Markov process that defines a solution the invariant measures are stable enough that there is relaxation to the unique equilibrium.

Blow-up and singularities. Our analysis shows that local solutions are smooth, although we do not control the lifespan of the solution. The mathematical results known so far do not rule out the emergence of singularities. This question is wide open, but some analytic results show that there are quite strong limitations on the type of blow up. We conjecture that if there is a blow up, most likely it will be a logarithmic pole in the sharp valleys.

In contrast, many numerical experiments do not report any problems of blow up, see Hoppe \& Nash [60,59] or [89, 87]. Numerical experiments from Blömker, Gugg \& Raible [12] furthermore indicate a fast convergence of spectral Galerkin methods for statistical quantities like the averaged surface roughness for the stochastic PDE.

There are also unpublished numerical results by Michael Winkler that seem to indicate blow-up behaviour for the deterministic equations with an additional strong linear instability, that is a lower order term which should not influence the question of existence and uniqueness. On the contrary Nolde et al. [15] are working on numerical verification of uniqueness, and their preliminary results look promising. These are based on Robinson et al. [24,34] or related results by Morosi \& Pizzocchero [76-78]. The method though only works for given fixed initial conditions or a fixed small ball of initial conditions. There are strong hints, see [97] for a general result and heuristic considerations, that additive noise should not be effective in preventing blow-up when this already happens in the deterministic equation.

Energy estimates are not sufficient to prevent blow-up. Recently, there has been interest in finding solutions for modified Navier-Stokes equations that do blow up by failing to be regular. Tao [113] constructed a modified nonlinearity that exhibits the same energy estimate than 3D-Navier-Stokes, but does admit solutions that blow up in finite time. A different approach in that spirit was presented by Li \& Sinai [71,72]. They constructed complex valued solutions of the Navier-Stokes equation that remain bounded in $L^{2}$ but blow up in finite time. The key idea in all these approaches is that due to the structure of the nonlinearity in Fourier space the mass is transported faster to higher Fourier modes than it is absorbed by dissipation. Thus leading to a blow up of regularity, when a non-trivial portion of the mass reaches infinity in finite time. In Section 7 we present a simplified result for our equation, proving that many complex-valued solutions blow up in finite time. This is somewhat not surprising, as the standard $L^{2}$-energy estimate fails to be true for complex valued solutions, however the lower bound derived in the proof of this result does not blow up in $L^{2}$. 
The randomly forced problem in the physical dimension two. When we consider the problem in dimension two, the regularity of initial conditions is not the only issue, as the weak regularity of the forcing starts playing a crucial role. Thus the difficulty due to the presence of noise is the lack of regularity of solutions. In dimension $d=1$ this is not an issue, as we expect solutions of the stochastic PDE to be continuously differentiable with respect to space and continuous in time, but in $d=2$ the solution fails to be that regular. As a consequence the square of the gradient $|\nabla h|^{2}$ in the nonlinearity is not defined directly.

A general solution is to rely on the the concept of regularity structures introduced by Martin Hairer [53], which was awarded the Fields medal recently, or the significantly simpler but less powerful approach of paraproducts and controlled distributions introduced by Gubinelli, Imkeller \& Perkowski [50]. In order to highlight the key problems we present in Section 4 the results of [18], based on results in [29], that apply similar ideas to our equation. This is the simplest case of the approach of Hairer [52], or Gubinelli et al. is effective. Here we do need to give sense of the quadratic nonlinearity for Gaussian random variables that are not regular enough to define the nonlinearity directly. On the other hand the other terms appearing in the proof are regular enough so that there is no trouble in their definition.

\subsection{The model}

Let us give more details about the model under consideration. We study the following equation for the graph of a growing surface

$$
\partial_{t} h=-\Delta^{2} h-\Delta|\nabla h|^{2}+\xi
$$

subject to periodic boundary conditions on the domain $[0, L]^{d}$ (i.e., $h$ and all its derivatives are $L$-periodic). The function $h(t, \cdot)$ models a height profile at time $t>0$, so $d=1$ and $d=2$ are the physically relevant dimensions. The noise $\xi$ models always space-time white noise, for instance due to fluctuations in the incoming gas of particles. We comment later in more detail on the mathematical model of the noise, which is given by the generalized derivative of a suitable Wiener process in Hilbert-space.

The equation is simplified here in the sense that lower order terms are left out, which do not present any obstacle in the theory of local existence and uniqueness, although some might cause problems for global estimates in the proof of global existence. We will discuss on possible additional terms in more detail in the next section.

Also for simplicity of presentation we consider only the rescaled version with nondimensional length-scales. We can rescale the variables $t, x$, and $h$ in order to move all relevant physical constants into the length of the domain $L$.

Remark 1 (Mass conservation) Due to the fact that the integral over a Laplacian vanishes for periodic boundary conditions, the deterministic equation conserves the total mass. Thus we always assume a moving frame condition

$$
\int_{[0, L]^{d}} h(t, x) d x=0 .
$$

To be more precise, the mean of $h$ for solutions of (1) decouples from the equation and the spatial average of the solution is just the spatial average of the noise, which behaves like a Brownian motion. Thus for simplicity we always consider a moving frame and massconservative noise by just subtracting the spatial mean of the noise in (1). Moreover, by a similar transformation one removes any constant growth rate of the surface from the equation. 

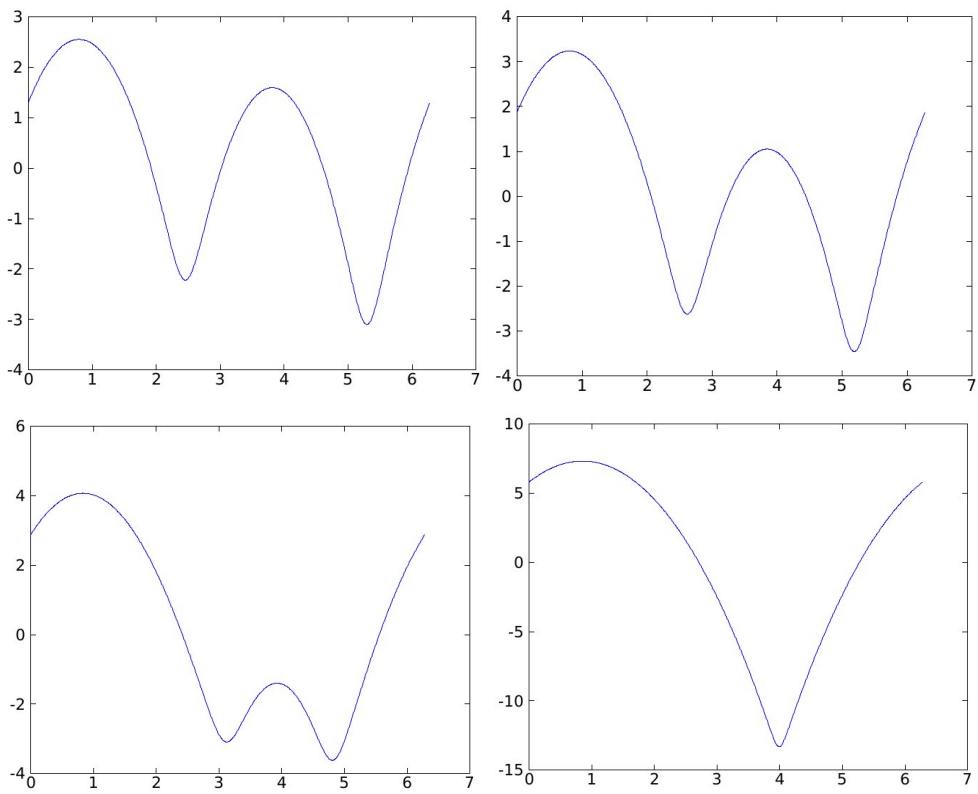

Fig. 1 Four snapshot at time $0.2,0.3,0,4$, and 0.7 of a numerical solution of the surface growth equation on $[0,2 \pi]$ with additional linear instability $-10 \partial_{x}^{2} h$. The hills look like parabolas with sharp valleys in between.

Remark 2 (Boundary Conditions) Although the surface is in experiments not periodic, these boundary conditions together with the assumption of a moving frame became the standard conditions in the physics literature for models of this type, as the effect of boundary condition is usually neglected in experiments. The surface is grown on large domains, and only small interior regions of almost periodic pattern are then measured. Sometimes the model has been considered also on the whole real line without decay condition at infinity, nevertheless we do not examine this case here in detail, as space-time white noise would make the solution at any time unbounded for $|x| \rightarrow \infty$.

From a mathematical point of view Neumann boundary conditions are quite similar for the problem (1) studied here, as they allow for similar energy estimates.

\subsection{Related Models}

In our presentation we ignore lower order terms, as we are after possible uniqueness or nonuniqueness. The main terms for questions about existence and regularity are the dominant fourth order linear operator $\Delta^{2} h$ and the quadratic non-linearity $\Delta|\nabla h|^{2}$.

Examples of lower order terms present in the models are the Kuramoto-Sivashinsky term $|\nabla h|^{2}$, which seems to be responsible for a saturation of hill sizes during coarsening, or a linear instability given by $-\Delta h$, which triggers an initial instability of a flat surface leading the the formation of hills. Both lower order terms are quite important for the dynamical behaviour, but not for questions regarding regularity and blow up. Moreover, the presence of these terms complicates calculations significantly (see [12] for an example). 
In order to take into account $-\Delta h$ in bounds uniform in time that yield global existence in $d=1$, gauge functions have to be used, as in Blömker \& Hairer [13]. See also Collet et al. [26], or Temam [115]. These yield estimates relative to a fixed surface (the gauge function) and its translations. But this is quite technical and up to now in our model it is only applied to Neumann boundary conditions. For periodic boundary conditions this question is still open.

There is also the choice of adding an additional cubic term $\nabla \cdot\left(|\nabla h|^{2} \nabla h\right)$ that does not change the scaling properties of the equation and that, equipped with the right sign, gives additional $L^{4}\left([0, T], W^{1,4}\right)$ regularity in a-priori estimates and even simplifies the proof of existence and uniqueness of global solutions. See for example Agélas [2].

An interesting nonlinearity that obeys the same symmetries as the terms present in (1) is $\operatorname{det}(\operatorname{Hess}(h))$. This nonlinearity allows for a gradient flow structure for the equation $[37,36]$

$$
\partial_{t} h=-\Delta h-\operatorname{det}(\operatorname{Hess}(h))
$$

with energy of the type $\int \Delta h|\nabla h|^{2} d x$, which is not bounded from below. Here Escudero, Gazzola \& Peral [36] showed that this nonlinearity alone leads to a blow up in finite time. Thus the conjecture is that adding additive noise will eventually always lead to a blow up, see [97] for related ideas. The combination of both nonlinearities destroys the gradient structure and nothing is known about the dynamics.

Let us briefly comment on some well studied related models:

1. The Kadar-Parisi-Zhang (KPZ) equation is [62]

$$
\partial_{t} h=\Delta h+|\nabla h|^{2}+\xi .
$$

For the deterministic equation, there is no problem with existence and uniqueness and via the Cole-Hopf transform $u=\mathrm{e}^{h}$ one can transform it to a linear heat equation. The stochastic equation is the toy-model for the theory of regularity structures [52,53], as already in $d=1$ solutions are not regular enough to define the nonlinearity in a trivial way. Here a renormalized nonlinearity has to be taken into account that takes care of divergent constants. This is different from the nonlinearity in (1), as here the infinite constant of the renormalization is eliminated by the additional Laplacian.

2. The Kuramoto-Sivashinsky equation is

$$
\partial_{t} h=-\Delta^{2} h-\Delta h+|\nabla h|^{2}+\xi .
$$

The deterministic equation (as well as the stochastic equation) for $d=1$ has no problem with local and global existence and uniqueness although the dynamics seems to be much richer than for (1). But for $d=2$ there is a lack of global estimates that would ensure global existence. Moreover, for the stochastic equation for $d=2$ the noise is too rough, and one needs to consider regularity structures and a renormalized nonlinearity, in order to define the equation.

3. Of course also the Navier-Stokes equation has many similarities, and many methods that work for the other equations were originally developed for Navier-Stokes. But as the type of equation is different, we do do give it in full details here.

\subsection{A-priori Estimates (1D-deterministic)}

As already pointed out, for global existence we heavily rely on the availability of an energy estimate. The key point for $d=1$ is a suitable cancellation of the non-linearity, namely using 
integration by parts

$$
\int_{0}^{L} h \partial_{x}^{2}\left(\partial_{x} h\right)^{2} d x=\frac{1}{3} \int_{0}^{L} \partial_{x}\left(\partial_{x} h\right)^{3} d x=0
$$

This is the main (and probably only) ingredient to derive useful a-priori estimates. Unfortunately, it already fails for $d=2$. Formally multiplying the deterministic equation by $h$ and integrating with respect to $x$, we obtain using (2),

$$
\partial_{t}\|h(t)\|_{L^{2}}^{2}+2\left\|\partial_{x}^{2} h(s)\right\|_{L^{2}}^{2} \leq 0 \quad \text { and } \quad\|h(t)\|_{L^{2}}^{2}+2 \int_{0}^{t}\left\|\partial_{x}^{2} h(s)\right\|_{L^{2}}^{2} d s \leq\|h(0)\|_{L^{2}}^{2} .
$$

Thus, by Poincaré inequality and Gronwall's Lemma,

$$
\|h(t)\|_{L^{2}} \leq \mathrm{e}^{-c t}\|h(0)\|_{L^{2}}^{2} \quad \text { and } \quad \int_{0}^{\infty}\|h(t)\|_{H^{2}}^{2} d t \leq\|h(0)\|_{L^{2}}^{2}
$$

As explained before this estimate is only valid for smooth local solutions up to a possible blow up, or one could use a spectral Galerkin approximation to verify it for global solutions. Note that the regularity implied by this estimate is enough for proving existence of solutions (see Section 5), but is lower than the critical regularity, that is, the minimal amount of regularity that would ensure uniqueness (see Section 3 ). In other words, the above regularity properties are super-critical with respect to the scaling invariance of the equation.

These estimates extend to the stochastic equation by a standard transformation. See Section 2.4 below for details. Let us remark that for the stochastic case it is even possible to bound exponential moments of the $L^{2}$-norm. See [38,106].

\subsection{A Lyapunov-type functional}

There is another a-priori estimate for smooth local solutions, which was helpful for $d=2$, but unfortunately it does not hold for spectral Galerkin method or any other approximation using projections,

$$
\begin{aligned}
\frac{1}{\alpha^{2}} \frac{d}{d t} \int_{0}^{L} \mathrm{e}^{\alpha h} d x & =\int_{0}^{L} \mathrm{e}^{\alpha h} h_{x} h_{x x x} d x+2 \int_{0}^{L} \mathrm{e}^{\alpha h} h_{x}^{2} h_{x x} d x \\
& =-\int_{0}^{L} \mathrm{e}^{\alpha h} h_{x x}^{2} d x+(2-\alpha) \int_{0}^{L} \mathrm{e}^{\alpha h} h_{x}^{2} h_{x x} d x \\
& =-\int_{0}^{L} \mathrm{e}^{\alpha h} h_{x x}^{2} d x-\frac{1}{3}(2-\alpha) \alpha \int_{0}^{L} \mathrm{e}^{\alpha h} h_{x}^{4} d x .
\end{aligned}
$$

Thus, for $\alpha \in(0,2)$,

$$
\int_{0}^{L} \mathrm{e}^{\alpha h(t)} d x \leq \int_{0}^{L} \mathrm{e}^{\alpha h(0)} d x \text { for all } t>0
$$

and

$$
\frac{(2-\alpha)}{3} \alpha^{3} \int_{0}^{\infty} \int_{0}^{L} \mathrm{e}^{\alpha h} h_{x}^{4} d x d t+\alpha^{2} \int_{0}^{\infty} \int_{0}^{L} \mathrm{e}^{\alpha h} h_{x x}^{2} d x d t \leq \int_{0}^{L} \mathrm{e}^{\alpha h(0)} d x .
$$

With some more effort (see Stein \& Winkler [108]) one can see that these terms are bounded independently of $h(0)$ for large $t$. 
The positive part $h^{+}=\max \{0, h\}$ now has much more regularity than the negative part $h^{-}=\max \{0, h\}$, so a possible blow up seems to be more likely to $-\infty$ than to $+\infty$. Unfortunately, this regularity is still not sufficient for uniqueness of solutions. It is just below the critical $B_{\infty}^{0, \infty}$-regularity. See Section 3 .

Nevertheless, this estimate was the key in [120] to prove the existence of global solutions in dimension $d=2$ using a discretization in time as the approximation method.

\subsection{Notation}

Let us finish the introduction by introducing some notation and properties used throughout the paper. We denote by $\Delta_{\text {per }}$ the Laplacian subject to periodic boundary conditions, and by $A=-\Delta_{\text {per }}^{2}$ the biharmonic operator. Its analytic semigroup is denoted by $S(t)=\mathrm{e}^{t A}$ for $t \geq 0$.

Let $\mathscr{D}^{\infty}$ be the space of infinitely differentiable $L$-periodic functions on $\mathbf{R}$ with zero mean in $[0, L]^{d}$. Likewise denote by $C_{\mathrm{per}}^{k}$ the space of $L$-periodic functions with zero mean that are $k$-times continuously differentiable. Define for $p \in[1, \infty]$

$$
\mathscr{L}^{p}=\left\{h \in L^{p}\left([0, L]^{d}\right): \int_{[0, L]^{d}} h(x) d x=0\right\} .
$$

with the standard $L^{p}$-norm. Here $\mathscr{L}^{2}$ is a Hilbert-space with norm and scalar product

$$
\|f\|_{\mathscr{L}^{2}}^{2}=\int_{[0, L] d}|f(x)|^{2} d x \quad \text { and }\langle f, g\rangle_{\mathscr{L}^{2}}=\int_{[0, L] d} f(x) g(x) d x .
$$

Let $\left(e_{k}\right)_{k \in \mathbf{N}_{\star}^{d}}$ be the orthonormal basis of $\mathscr{H}=\mathscr{L}^{2}$ given by products of the trigonometric functions $\sin \left(2 m_{i} \pi x_{i} / L\right)$ and $\cos \left(2 m_{i} \pi x_{i} / L\right)$ with $m \in \mathbf{N}_{\star}^{d}=\mathbf{N}^{d} \backslash\{0\}$, and let $\lambda_{k}$ be the eigenvalues of $A$ such that

$$
A e_{k}=\lambda_{k} e_{k}
$$

Notice that $\lambda_{k} \sim-|k|^{4}$. With a slight abuse of notation, we also denote the complex basis by $\left(e_{k}\right)_{k \in \mathbf{Z}_{\star}^{d}}$, which is given by the normalized $\mathrm{e}^{i 2 \pi k \cdot x / L}$ for $k \in \mathbf{Z}_{\star}^{d}=\mathbf{Z}^{d} \backslash\{0\}$.

The Sobolev spaces $\mathscr{H}^{\gamma}$ for $\gamma \in \mathbf{R}$ are defined as the domains of fractional powers of $-A$, which are equivalent to standard Sobolev spaces (up to the zero-mean constraint). See for example Henry [57], Pazy [83], or Lunardi [75]. Here we use the explicit expansions of norms in term of Fourier series

$$
\mathscr{H}^{\gamma}=\left\{u=\sum_{k \in \mathbf{N}_{\star}^{d}} \alpha_{k} e_{k}:\|u\|_{\mathscr{H}^{\gamma}}^{2}=\sum_{k \in \mathbf{N}_{\star}^{d}} \alpha_{k}^{2}\left|\lambda_{k}\right|^{\gamma / 2}<\infty\right\} .
$$

This is equivalent to saying that $\mathscr{H}^{\gamma}$ consist of all functions $h$ in $H_{l o c}^{\gamma}\left(\mathbf{R}^{d}\right)$ which are $L$ periodic and satisfy $\int_{[0, L]^{d}} h(x) d x=0$. Furthermore, the standard $H^{\gamma}\left([0, L]^{d}\right)$-norm is an equivalent norm on $\mathscr{H}^{\gamma}$. We also use the space

$$
\mathscr{W}^{1,4}:=\left\{u \in \mathscr{H}^{1}:\|u\|_{\mathscr{W}^{1,4}}=\|u\|_{\mathscr{L}^{4}}+\|\nabla u\|_{\mathscr{L}^{4}}<\infty\right\} .
$$

Note that all usual Sobolev embeddings, such as for $d=1$ for example $\mathscr{H}^{1} \subset C^{0}([0, L])$ or $\mathscr{H}^{2} \subset \mathscr{W}^{1,4}$ still hold. See [99] or [1] for details on Sobolev spaces.

The nonlinearity is throughout the paper denoted by

$$
B(u, v)=\Delta(\nabla u \cdot \nabla v) .
$$

This is a bilinear map, which is defined for all $u, v \in \mathscr{H}^{1}$ with values in a $\mathscr{H}^{\gamma}$ with $\gamma$ sufficiently negative. We have the following bounds for $d=1,2$ : 
Lemma 1 If $\alpha, \beta, \gamma \geq 0$ and $\alpha+\beta+\gamma \geq d / 2$ (with strict inequality if at least one of the numbers is equal to $d / 2$ ), then B maps $\mathscr{H}^{1+\alpha} \times \mathscr{H}^{1+\beta}$ continuously into $\mathscr{H}^{-2-\gamma}$. In particular, there exists $c=c(\alpha, \beta, \gamma)$ such that

$$
\left\|B\left(u_{1}, u_{2}\right)\right\|_{\mathscr{H}^{-2-\gamma}} \leq c\left\|u_{1}\right\|_{\mathscr{H}^{1+\alpha}}\left\|u_{2}\right\|_{\mathscr{H}^{1+\beta}} .
$$

Proof Explicit calculation using Fourier series or abstract $\mathscr{H}^{\delta}$ bounds on products of $\mathscr{H}^{\alpha}$ and $\mathscr{H}^{\beta}$ in the space $\mathscr{H}^{-\gamma}$ [99]. For $d=2$ see [18, Lemma A.1] for $d=1$ see [19].

The following Lemma is necessary for $d=2$ when one $\operatorname{argument}$ in $B$ fails to have a gradient.

Lemma 2 Fix dimension $d=2$. Let $\varepsilon \in(0,1)$ and $\gamma>0$. For every $\alpha \in(0,1)$ and $q>2$ such that $1-\alpha+\frac{2}{q}<\varepsilon \wedge \gamma$, there is a constant $c>0$ such that

$$
\left\|B\left(u_{1}, u_{2}\right)\right\|_{\mathscr{H}^{-2-\gamma}} \leq c\left\|u_{1}\right\|_{W^{\alpha, q}}\left\|u_{2}\right\|_{\mathscr{H}^{1+\varepsilon}}, \quad \text { for every } u_{1} \in W^{\alpha, q} \text { and } u_{2} \in \mathscr{H}^{1+\varepsilon} \text {. }
$$

Proof Straightforward. See [18, Corollary A.3] or Sobolev space bounds for products of distributions and regular functions in [99].

Note that the conditions on $\alpha, \gamma$, and $\varepsilon$ in the Lemma above are sufficient for the results presented here, but could be relaxed by using the paracontrolled approach.

\section{Brief introduction to SPDE}

Here we give a brief self-contained introduction to SPDEs treating only the case of additive noise needed for our model. For details on stochastic partial differential equations (SPDEs) we refer to [31,25,73,51,85,116], the recent second edition of DaPrato \& Zabczyk [32], or the review article [106].

In a formal way (using $\xi=\partial_{t} W$ and multiplying by $d t$ ) we can rewrite (1) as an abstract stochastic evolution equation

$$
d h=(A h+B(h, h)) d t+d W
$$

where $W$ is a suitable $\mathscr{Q}$-Wiener process, which we define below. Moreover, we need to give a rigorous meaning to the formal Equation (4).

Let us remark that for stochastic PDEs on unbounded domains one can use the formulation of Walsh [118], although one has to consider that for space-time white noise both the stochastic convolution $Z(t, x)$ and its derivative $\nabla Z(t, x)$ (if it exists) are unbounded for $|x| \rightarrow \infty$. We avoid these technicalities here, by considering periodic boundary conditions only.

Let us first give the abstract definition of a $\mathscr{Q}$-Wiener process $W$ and later give an explicit representation in terms of Fourier series. Fix for the whole paper a probability space $(\Omega, \mathscr{A}, \mathbb{P})$.

Definition 1 (Q2-Wiener process) Given a symmetric, non-negative, and continuous operator $\mathscr{Q}$ on $\mathscr{H}$, we call a $\mathscr{H}$-valued stochastic process $\{W(t)\}_{t \geq 0}$ a $\mathscr{Q}$-Wiener process, if

- $W(0)=0(\mathbb{P}$-almost sure $)$

- W has (P-almost sure) continuous trajectories (or paths): $\left\{\begin{aligned} {[0, \infty) } & \rightarrow \mathscr{H} \\ t & \mapsto W(t)\end{aligned}\right.$ 
- The increments of $W$ are stochastically independent, i.e. the random variables

$$
W\left(t_{1}\right), W\left(t_{2}\right)-W\left(t_{1}\right), \ldots, W\left(t_{n}\right)-W\left(t_{n}-1\right)
$$

are independent for any choice of $n \in \mathbf{N}$ and $0 \leq t_{1} \leq t_{2} \leq \ldots \leq t_{n}$

- The increments are Gaussian with $W(t)-W(s) \sim \mathcal{N}(0,(t-s) \mathscr{Q})$ for all $0 \leq s \leq t$

Note that in case $\mathscr{H}=\mathbb{R}$ the covariance operator $\mathscr{Q}=1$ is just a number, and the Wiener process is a real-valued standard Brownian motion. See [101,79,63] for properties.

In the definition above we used the notation $X \sim \mathscr{N}(0, \Sigma)$ for a $\mathscr{H}$-valued random variable $X$ and a non-negative symmetric operator $\Sigma$ on $\mathscr{H}$, which means that for all testfunctions $\varphi$ in $\mathscr{H}$ the real-valued random variable $\langle X, \varphi\rangle$ is Gaussian (i.e. normal) distributed with

$$
\text { expectation } \quad \mathbb{E}\langle X, \varphi\rangle=0 \quad \text { and variance } \quad \mathbb{E}\langle X, \varphi\rangle^{2}=\langle\Sigma \varphi, \varphi\rangle .
$$

Moreover, we have the covariance

$$
\mathbb{E}\langle X, \varphi\rangle\langle X, \psi\rangle=\langle\Sigma \varphi, \psi\rangle \text { for all } \varphi, \psi \in \mathscr{H} .
$$

In the following we will always consider $\mathscr{Q}: \mathscr{L}^{2} \rightarrow \mathscr{L}^{2}$ to be a bounded symmetric linear operator such that

$$
\mathscr{Q} e_{k}=\alpha_{k}^{2} e_{k}, \quad k \in \mathbf{N}_{\star}^{d},
$$

so that $\mathscr{Q}$ is non-negative. This is sufficient to model all kinds of spatially homogeneous Gaussian noise $\xi=\partial_{t} W$ such that formally

$$
\mathbb{E} \xi(t, x)=0 \quad \text { and } \quad \mathbb{E} \xi(t, x) \xi(s, y)=\delta(t-s) q(x-y),
$$

where $q$ is the the spatial correlation function (or distribution). Now $\mathscr{Q}=q \star$, which is the convolution operator with $q$. For details see [8], the references therein, and also [32, Sec. 4.15].

Note that mass-conservation is already built into the model of the noise by the choice of $\mathscr{H}$.

Remark 3 A Wiener process is not uniquely defined. From the definition it is easy to check, that it exhibits self-similarity and shift invariance:

- $\delta\left\{W\left(\delta^{-2} t\right)\right\}_{t \geq 0}$ is a $\mathscr{Q}$-Wiener process for all $\delta>0$.

- $\{W(t+s)-W(s)\}_{t \geq 0}$ is a $\mathscr{Q}$-Wiener process for all $s>0$.

We call an I-Wiener process a standard cylindrical Wiener-process. Obviously, this exists only in a generalized sense, as $W(t) \notin \mathscr{H}$. This follows from the fact that for any $\mathscr{Q}$-Wiener process a direct calculation shows $\mathbb{E}\|W(t)\|^{2}=\operatorname{trace}(\mathscr{Q})$, together with Fernique's theorem ([32, Sec. 2.21]) stating that $\mathscr{H}$-valued Gaussian random variables have all and especially second moments.

For a cylindrical Wiener process, one can show from the definition that for any orthonormal basis $\left\{f_{k}\right\}_{k \in \mathbb{N}}$ of smooth functions the random variables $\left\langle W(\cdot), f_{k}\right\rangle$ are a family Brownian motions. Additionally, one can show that they are stochastically independent [32, Prop. 4.3]. Moreover,

$$
\langle W(t), \varphi\rangle=\sum_{k=1}^{\infty}\left\langle W(t), f_{k}\right\rangle\left\langle\varphi, f_{k}\right\rangle
$$

is a well defined real-valued Gaussian with mean 0 variance $\|\varphi\|^{2}$ and covariance $\langle\varphi, \psi\rangle$. 
Example 1 For a sequence of independent identically distributed (i.i.d.) standard Brownian motions $\left\{\beta_{k}\right\}_{k \in \mathbf{N}}$, an orthonormal basis $\left\{f_{k}\right\}_{k \in \mathbf{N}}$ of $\mathscr{H}$, and $\left\{\alpha_{k}\right\}_{k \in \mathbf{N}} \subset \mathbf{R}$ such that $\sum_{k \in \mathbf{N}} \alpha_{k}^{2}<\infty$ define

$$
W(t)=\sum_{k \in \mathbf{N}} \alpha_{k} \beta_{k}(t) f_{k}
$$

Then $W$ is a $\mathscr{Q}$-Wiener process with $\mathscr{Q} e_{k}=\alpha_{k}^{2} f_{k}$.

Conversely, any $\mathscr{Q}$-Wiener process can be written in this series expansion [32, Prop. 4.3], where $f_{k}$ is an orthonormal basis of eigenfunctions of $\mathscr{Q}$ with corresponding eigenvalues $\alpha_{k}$. Moreover, if the $\alpha_{k}$ are only bounded, we obtain a cylindrical Wiener process.

Remark 4 One could assume in the Definition that the Wiener-process is Hölder continuous in time for any exponent below $\frac{1}{2}$, but we do not need this here.

2.1 Regularity of the stochastic convolution

We consider a standard cylindrical Wiener process and expand it with respect to the eigenfunctions $\left\{e_{k}\right\}_{k \in \mathbf{N}_{\star}^{d}}$, taking into account the mass-conservation, i.e. for a family of i.i.d. standard Brownian motions $\left\{\beta_{k}\right\}_{k \in \mathbf{N}_{\star}^{d}}$ we have

$$
W(t)=\sum_{k \in \mathbf{N}_{\star}^{d}} \beta_{k}(t) e_{k} .
$$

Define the corresponding Ornstein-Uhlenbeck process for $t>0$ as the following Itō-integral:

$$
Z(t)=\int_{0}^{t} \mathrm{e}^{(t-s) A} d W=\sum_{k \in \mathbf{N}} \int_{0}^{t} \mathrm{e}^{(t-s) \lambda_{k}} d \beta_{k}(t) e_{k} .
$$

This can be defined path-wise without Itō-calculus using the integration by parts formula:

$$
Z(t)=W(t)+A \int_{0}^{t} \mathrm{e}^{(t-s) A} W(s) d s .
$$

Note that $Z$ solves formally

$$
\partial_{t} Z+\Delta^{2} Z=\partial_{t} W \quad \text { with } Z(0)=0 .
$$

Let us first show that $Z$ is not too regular in space:

Lemma 3 For every $t>0$,

$$
\mathbb{E}\left[\|Z(t)\|_{\mathscr{H}^{2-d / 2}}^{2}\right]=\infty
$$

and $Z \notin \mathscr{H}^{2-d / 2}$ for all times, almost surely.

Proof Using the explicit representation of $Z$ in Fourier series and Itō-isometry, yields

$$
\mathbb{E}\left[\|Z(t)\|_{\mathscr{H}^{2-d / 2}}^{2}\right]=\sum_{k \in \mathbf{N}_{\star}^{d}}|k|^{4-d} \int_{0}^{t} \mathrm{e}^{2 \lambda_{k}(t-s)} d s \leq C \sum_{k \in \mathbf{N}_{\star}^{d}} \frac{1}{2|k|^{d}}\left(1-\mathrm{e}^{2 \lambda_{k} t}\right)=\infty .
$$

The almost sure statement follows from Gaussianity. See for example [20, Theorem 2.5.5]. The key point is due to Fernique's theorem: The probability of $Z$ being in a given Banach space is positive if and only if $Z$ has some exponential moments in this space. 
Lemma 3 above shows for example that for $d=2$ the gradient of $Z$ is not defined as a function. On the other hand, $Z$ has a "fractional" derivative of all orders smaller than $2-d / 2$ not only in $L^{2}$ but also in all $L^{p}$.

Proposition 1 For every $p \geq 1$ and $s \in(0,2-d / 2)$,

$$
\sup _{t>0} \mathbb{E}\left[\|Z(t)\|_{W^{s, p}}^{p}\right]<\infty \text {. }
$$

Proof We rely on the explicit representation of the norm [99, Subsec. 2.2.4 \& 2.4.4] for $s \in(0,1)$. For $d=1$ and $s \in\left(1, \frac{3}{2}\right)$ we need to consider the regularity of $\partial_{x} Z(t)$ in $W^{s, p}$.

$$
\|z\|_{W^{s, p}}^{p}=\|z\|_{\mathscr{L}^{p}}^{p}+\int_{[0, L]^{d}} \int_{[0, L]^{d}} \frac{|z(x)-z(y)|^{p}}{|x-y|^{d+s p}} d x d y .
$$

Use the Fourier representation of $Z$ with $Z_{k}=\left\langle Z, e_{k}\right\rangle$ to get,

$$
\mathbb{E}\left[|Z(t, x)-Z(t, y)|^{2}\right] \leq c \sum_{k \in \mathbf{N}_{\star}^{d}} \mathbb{E}\left[\left|Z_{k}(t)\right|^{2}\right]\left|e_{k}(x)-e_{k}(y)\right|^{2} \leq c \sum_{k \in \mathbf{N}_{\star}^{d}} \frac{1 \wedge|k \cdot(x-y)|^{2}}{|k|^{4}}
$$

Now for any $\gamma \in(s, \min \{1,2-d / 2\})$ we find a constant such that

$$
\mathbb{E}\left[|Z(t, x)-Z(t, y)|^{2}\right] \leq c \sum_{k \in \mathbf{N}_{\star}^{d}}|k|^{2 \gamma-4}|x-y|^{2 \gamma} \leq C|x-y|^{2 \gamma}
$$

By Gaussianity, for every $p \geq 1, \mathbb{E}\left[|Z(t, x)-Z(t, y)|^{p}\right] \leq c_{p}|x-y|^{p \gamma}$. Therefore,

$$
\mathbb{E}\left[\iint \frac{|Z(t, x)-Z(t, y)|^{p}}{|x-y|^{2+s p}} d x d y\right] \leq C \iint|x-y|^{-d+p(\gamma-s)} d x d y<\infty .
$$

The missing cases and the $\mathscr{L}^{p}$-norm are bounded in a similar way.

Remark 5 (Continuity in time) The regularity in time from the previous proposition is only $L^{p}$, but we can improve this, with standard arguments, to $L^{\infty}$ (using the factorization method) or even Hölder continuity (using also fractional norms in time). For details we refer to the methods introduced in [31], see for example [28].

\subsection{Mild formulation}

As solutions of SPDEs are usually not regular enough to give a meaning to (4), the solution is interpreted in some weaker sense. One possibility is the mild formulation given by the variation of constants formula:

Definition 2 (Mild Solution) We call a continuous stochastic process $h \in C^{0}\left(\left[0, \tau^{*}\right), \mathscr{H}^{\zeta}\right)$ for some almost sure positive stopping time $\tau^{*}>0$ a mild solution of (4) with initial condition $h_{0}$ if

$$
h(t)=\mathrm{e}^{t A} h_{0}+\int_{0}^{t} \mathrm{e}^{(t-s) A} B(h(s), h(s)) d s+Z(t)
$$

for all $t \in\left(0, \tau^{*}\right) \mathbb{P}$-almost surely.

We call a mild solution (strongly) unique, if for a given $h_{0}$ and fixed process $Z$ any two mild solutions coincide up to the minimum of their stopping times. 
Now the main problem in this setting is to determine the regularity of $Z$, which can be read in terms of the covariance of the driving noise. Once we know this, we can solve the equation using Banach's fixed point argument.

Clearly mild solutions can be defined on different spaces than $C^{0}\left(\left[0, \tau^{*}\right), \mathscr{H}^{\zeta}\right)$, especially with weaker regularity at $t=0$. The definition given above can be easily modified for these different settings without serious difficulties, so we will not provide further details and will keep using the notion of "mild solution".

\subsection{Weak formulation}

Another concept of solutions is the weak formulation, where (4) is integrated against smooth functions. Recall first the following result, an easy consequence of the Poincare inequality, which ensures that all integrals in (7) are well defined.

Lemma 4 If $u$ is a distribution on $\mathbf{R}^{d}$ such that $\nabla u \in L_{\mathrm{loc}}^{2}\left([0, \infty), \mathscr{L}^{2}\right)$, then $u \in L_{\mathrm{loc}}^{2}\left([0, \infty), \mathscr{L}^{2}\right)$ and thus $u \in L_{\text {loc }}^{2}\left([0, \infty), \mathscr{H}^{1}\right)$.

A weak solution for (1) with initial condition $h_{0} \in \mathscr{L}^{1}$ is any distribution $h$ with locally square integrable gradient $\nabla h \in L_{\text {loc }}^{2}\left([0, \infty), \mathscr{L}^{2}\right)$ such that for every smooth function $\phi$, which is compactly supported in time and $L$-periodic in space, i.e. $\phi \in C_{c}^{\infty}\left([0, \infty), \mathscr{D}^{\infty}\right)$,

$$
\begin{aligned}
\int_{0}^{\infty} \int_{[0, L]^{d}} h(t, x) \partial_{t} \phi(t, x) d x d t & \\
-\int_{0}^{\infty} \int_{[0, L]^{d}} & {\left[h(t, x) \Delta^{2} \phi(t, x)+|\nabla h(t, x)|^{2} \Delta \phi(t, x)\right] d x d t } \\
& =-\int_{[0, L]^{d}} h_{0}(x) \phi(0, x) d x+\int_{0}^{\infty} \int_{[0, L]^{d}} W(t, x) \partial_{t} \phi d x d t
\end{aligned}
$$

Lemma 4 ensures that all terms in the formula above are well defined. Moreover, the solution is only defined up to constants.

There are many other definitions of a weak solution imposing additional regularity or using test-functions only in space as in the following Proposition.

Proposition 2 (mild implies weak) Assume $Z$ is well defined in $\mathscr{H}$. Let $h$ be a mild solution in $\mathscr{H}$ as in Definition 2 with $\langle B(u, u), \varphi\rangle \in L^{1}\left(\left[0, \tau^{\star}\right]\right)$ for all $\varphi \in D(A)$, then $u$ is a weak solution. This means that for any $\varphi \in D(A)$ we have

$$
\left\langle h(t)-h_{0}-W(t), \varphi\right\rangle_{\mathscr{H}}=\int_{0}^{t}\langle h(s), A \varphi\rangle+\langle B(h(s), h(s)), \varphi\rangle d s
$$

Proof (Idea of Proof) The key idea of proof is to note that $\left\langle\mathrm{e}^{t A} u, A \varphi\right\rangle=\partial_{t}\left\langle\mathrm{e}^{t A} u, \varphi\right\rangle$. Then one needs to check that due to the additional regularity assumption all terms are well-defined and one can integrate by parts.

We remark, that the mild formulation in Proposition 2 is not the same as in (7), but one can derive (7) by considering first elementary step functions with values in $C_{\mathrm{per}}^{\infty}\left([0, L]^{d}\right)$ and then approximate $\phi$. 


\subsection{Transformation to a random PDE}

Indeed, using the standard method of looking at the difference $v=h-Z$ between the solution and the stochastic convolution, the stochastic PDE can be transformed into a random PDE.

$$
\partial_{t} v=A v+B(v, v)+2 B(v, Z)+B(Z, Z) .
$$

If the stochastic convolution $Z$ is sufficiently regular, then for each instance of chance the path-wise solvability for the SPDE is completely analogous to the deterministic results and one only needs to consider the two additional lower order terms $B(v, Z)$ and $B(Z, Z)$.

This covers many examples like our equation with $d=1$, but for the surface growth equation in $d=2$ the OU-process $Z$ is no longer regular enough to define $B(Z, Z)$. Moreover, we need more regularity of $v$, in order to define the product of $\nabla v$ with the distribution $\nabla Z$ in $B(Z, Z)$. See Section 4.

\section{Local Uniqueness}

In this section, following the technique introduced in the remarkable paper by Koch \& Tataru [65] on the Navier-Stokes equations, we review the results of [17] on local existence and uniqueness of solutions with initial data in the largest possible critical space where weak solutions make sense. This space contains all previous spaces where analogous results were proved. Let us remark that the same method has been applied to other fourth order problems $[66,119]$.

Our main motivation to study (1) in the largest possible space actually comes from the stochastically perturbed equation, where, due to noise, solutions are not regular enough, and we intend to use as least regularity as possible. However, for dimension $d=2$ this approach does not work. We comment later in Section 4 on this. In view of the transformation of Section 2.4 we consider first only the deterministic equation, and add sufficiently smooth noise later. Thus consider

$$
\partial_{t} h=A h+B(h, h), \quad h(0)=h_{0} .
$$

At least for dimension $d \leq 3$ the largest critical space of initial conditions introduced by Koch \& Tataru is in our setting equivalent to the homogeneous Besov space $\dot{B}_{\infty}^{0, \infty}$. This space is much simpler to work with, and it simplifies the results on existence and uniqueness. Moreover, we see in Section 3.6 that all such solutions are smooth in the space and time variable for $t>0$.

Remark 6 (Smooth initial conditions) For regular initial conditions, for example in $\mathscr{H}^{1}$ for $d=1$, standard arguments using a fixed point theorem in $C^{0}\left([0, T], \mathscr{H}^{1}\right)$ assure uniqueness of local solutions. Moreover, one can continue the solution in $\mathscr{H}^{1}$ until the norm blows up in that space. One can also verify that small initial conditions lead to the global existence of a small smooth solution for all times. Here we can go below that regularity for uniqueness of solutions. But we are still far from being able to prove uniqueness of global solutions in the general case, as they are bounded for $d=1$ only in $\mathscr{L}^{2}$.

Let us remark that global existence and uniqueness for small initial conditions was also established in [91] for multiplicative noise, at least with high probability. 


\subsection{Notion of critical spaces}

An account on the scaling heuristic for the Navier-Stokes equations can be found for example in Cannone [22], such arguments are the basis of the celebrated result on partial regularity of Caffarelli, Kohn, \& Nirenberg [21].

The rationale behind the method is the following. First, notice that (9) is on the whole $\mathbf{R}^{d}$ invariant under the scaling transformation

$$
h(t, x) \longrightarrow h_{\lambda}(t, x)=h\left(\lambda^{4} t, \lambda x\right)
$$

If $X$ is a functional space for $h$ (for example $L^{\infty}\left(0, \infty ; L^{2}\left(\mathbf{R}^{d}\right)\right)$ or time-independent like $H^{1}\left(\mathbf{R}^{d}\right)$ ), we can consider how the norm of $X$ scales with respect to the transformation (10) above. If

$$
\left\|h_{\lambda}\right\|_{X}=\lambda^{-\alpha}\|h\|_{X}
$$

then we can distinguish the three cases:

$$
\alpha<0, \text { sub-critical } \quad-\quad \alpha=0, \text { critical } \quad-\quad \alpha>0, \text { super-critical. }
$$

The super-critical case corresponds to small-scales behaviour and is related to low regularity, typically to topologies where possibly existence of weak solutions can be proved, but no regularity or uniqueness. In the super-critical case we can think of the nonlinearity of the problem as a non-negligible perturbation of the linear equation.

In the sub-critical case with sufficiently high regularity, it is possible to give good bounds on the nonlinearity and thus proceed with a fixed-point argument yielding uniqueness and existence at least locally. This is due to the fact that in this regime the nonlinearity of the problem is a perturbation of order smaller than the order of the linear part.

Due to scaling we can decrease the size of the initial conditions in the sub-critical case, thus we can rely on a result of the type: Small data implies global existence. Hence, the critical cases are the most interesting ones.

Consider spaces $X$ (depending on the space variable) and $Y_{T}$ (depending on both variables, with $t$ up to $T>0$ ), then in order to have a regularity criterion based on $Y_{T}$, the following statements must hold,

1. there is a unique local solution for every initial condition in $X$,

2. the unique local solution we have found is regular, and

3. the unique local solution can be continued up to time $T$, as long as its norm in $Y_{T}$ stays bounded.

The above analysis has been extensively carried out in numerous papers for the three dimensional Navier-Stokes equations (see for examples references in Cannone [22]). The first paper dealing with such aims were Prodi [86] and Serrin [103], see also Beale, Kato \& Majda [7].

We want to point out though that the scaling heuristic is an oversimplification where we look at the nonlinearity of the problem only in terms of its size. Tao [113] for instance considers a large class of problems that "look like" the Navier-Stokes equations in terms of their scaling and their continuity properties, and that develop singularities in finite time. On the other hand Barbato et al. [4] show global existence and uniqueness in the super-critical regime for the viscous dyadic Navier Stokes model. We finally notice, see [112,5,6], that at the critical level one can still allow for a super-critical logarithmic correction. 
3.2 Setting

As outlined in the previous section, the interesting norms are the critical ones that do not change under rescaling. Thus we consider our periodic solutions extended to $\mathbf{R}^{d}$ and use the scaling invariant version (under (10)) of the $L_{\text {loc }}^{2}$-space for the gradient $\nabla h$, defined by the following norm:

$$
\|h\|_{\mathscr{X}^{0}}:=\left(\sup _{x \in \mathbf{R}^{d}, R>0}\left\{\frac{1}{R^{d+2}} \int_{0}^{R^{4}} \int_{B_{R}(x)}|\nabla h|^{2} d y d t\right\}\right)^{\frac{1}{2}} .
$$

This definition follows the ideas of [65] and is the largest critical space in which weak solutions still are defined, as we need a locally square integrable gradient, in order to have the weak formulation in (7) defined. Our first aim is to state a simpler representation of the bi-caloric extension (see below) in this space.

Define therefore the linear space $\mathscr{X}$ of functions such that

$$
\|k\|_{\mathscr{X}}=\sup _{t>0}\left\{t^{\frac{1}{4}}\|\nabla k(t)\|_{\infty}\right\}<\infty .
$$

A local in time version of this space can be defined for any $R>0$ by

$$
\|k\|_{\mathscr{X}_{R}}:=\sup _{t \leq R^{4}}\left(t^{\frac{1}{4}}\|\nabla k(t)\|_{\infty}\right) .
$$

Note that we always identify functions that differ only by a constant. This is motivated by the fact that the equation is mass-conservative.

For an $L$-periodic function, we denote $\mathrm{e}^{t A} k$ as the bi-caloric extension of $k$. Note that the semigroup extended on the whole $\mathbf{R}^{d}$ is also given by the convolution with the Greens function $G(t, x)$, which has the Fourier transform (with respect to the space variable) $\widehat{G}(t, \xi)=$ $\mathrm{e}^{t|\xi|^{4}}$.

Denote by $\mathscr{B}$ the homogeneous Besov space $\dot{B}_{\infty}^{0, \infty}$, where the dot denotes mass-conservation. We use an equivalent norm on $\mathscr{B}$ given by the bi-caloric extension

$$
\|k\|_{\mathscr{B}}:=\left\|\mathrm{e}^{t A} k\right\|_{\mathscr{X}} .
$$

See for instance [69] for a characterization of Besov spaces in terms of heat kernels. Here we use the bi-Laplace operator $A$, which changes the scaling. We define similarly local versions $\mathscr{B}_{R}$.

The following proposition shows that the space $\mathscr{B}$ is actually equivalent to the caloric extension in the scale-invariant space $\mathscr{X}^{0}$.

Proposition 3 (Proposition 2.2. of [17]) Assume $d \leq 3$. Then $\mathrm{e}^{t A} k \in \mathscr{X}^{0}$ if and only if $k \in \mathscr{B}$ and the norm in $\mathscr{B}$ is equivalent to the norm defined by the bi-caloric extension in $\mathscr{X}^{0}$.

This means, that there are constants $c_{1}, c_{2}>0$ such that

$$
c_{1}\|k\|_{\mathscr{B}} \leq\left\|\mathrm{e}^{t A} k\right\|_{\mathscr{X}^{0}} \leq c_{2}\|k\|_{\mathscr{B}} .
$$

Proof (Idea of Proof) The key point is to prove (11). The inequality on the right holds in any dimension $d \geq 1$ since it is straightforward to check that $\|\cdot\|_{\mathscr{X} 0}$ is bounded by $\|\cdot\|_{\mathscr{X}}$. For the inequality on the left, one can use the explicit representation of the semigroup in terms of the Greens function. Moreover, by scaling and translations invariance, it is sufficient to show a bound on $t^{\frac{1}{4}}\left(\nabla \mathrm{e}^{t A} k\right)(x)$ for $t=1$ and $x=0$, only. This is then technically quite involved. For details see [17, p. 369]. 
3.3 Examples

The Besov space $\mathscr{B}$ is well understood, it contains for example the space $B M O$ of functions of bounded mean oscillations. In view of the fixed point argument of Theorem 1 we wish to recall the discussion of [17], that is for which initial conditions it is possible to find $R>0$ such that the initial condition is small in the $\mathscr{B}_{R}$ norm. For this aim define

$$
\mathscr{Z}=\left\{k: \mathbf{R}^{d} \rightarrow \mathbf{R}:\|k\|_{\mathscr{B}_{R}} \rightarrow 0 \text { as } R \downarrow 0\right\} .
$$

Functions in $\mathscr{Z}$ correspond to initial conditions where it is possible to solve the (9) locally in $\mathscr{B}_{R}$. In this section we give a few examples of subspaces of $\mathscr{Z}$. Then we show that bounded functions in $L^{\infty}\left(\mathbf{R}^{d}\right)$, although contained in $\mathscr{B}$, are not contained in $\mathscr{Z}$. Hence the method of proof for local uniqueness presented here fails for initial conditions in $L^{\infty}\left(\mathbf{R}^{d}\right)$ with large norm.

Lemma 5 The following statements hold,

- if $k: \mathbf{R}^{d} \rightarrow \mathbf{R}$ is bounded and uniformly continuous, then $k \in \mathscr{Z}$, i.e. the periodic extension of the space $\mathscr{D}^{\infty}$ is in $\mathscr{Z}$

- if $k: \mathbf{R}^{d} \rightarrow \mathbf{R}$ has bounded gradient on $\mathbf{R}^{d}$, then $k \in \mathscr{Z}$,

- the periodic extension of $\mathscr{H}^{d / 2}$ is in $\mathscr{Z}$.

The proof of this Lemma is established by direct calculations using the representation of the norms and the Greens function. See Lemma 3.1 of [17] for more details.

In the following we only give an example on the whole $\mathbf{R}^{d}$, but it is easy to extend this to the periodic case. Since the Green's function tensorizes, it is enough to find examples for $d=1$.

Example 2 There are functions in $L^{\infty}\left(\mathbf{R}^{d}\right)$ not belonging to $\mathscr{Z}$. Define $k(x)=\mathbb{1}_{[-1,1]}(x)$, then using the Green's function one can show $\|k\|_{\mathscr{B}_{R}} \geq G(1,0)>0$.

Example 3 The space $\mathscr{Z}$ contains unbounded functions, which are not in $\mathscr{B}$. Define for $\alpha>$ $0, k_{\alpha}(x)=|x|^{\alpha}$. Now one can show $\left\|t^{\frac{1}{4}} \partial_{x}\left(\mathrm{e}^{t A} k_{\alpha}\right)\right\|_{\infty}=t^{\frac{\alpha}{4}}\left\|\partial_{x}\left(\mathrm{e}^{A} k_{\alpha}\right)\right\|_{\infty}$. Hence, $\left\|k_{\alpha}\right\|_{\mathscr{B}_{R}} \rightarrow 0$ for $R \rightarrow 0$ but $\left\|k_{\alpha}\right\|_{\mathscr{B}}=\infty$.

Example 4 Consider the case $d=1$, then $k(x)=\log (|x|)$ is (pointwise, away from the pole) a stationary solution for problem (9). See Remark 14 or [16] for more examples like the periodic $\log (\sin (x))$. Nevertheless, this function is neither a weak nor a mild solution, as $\nabla k$ is not locally square summable.

Now it is possible to show that $k \in \mathscr{B}$ but $k \notin \mathscr{Z}$, as $\|k\|_{\mathscr{B}}$ is finite, but $\|k\|_{\mathscr{B}_{R}}$ is independent of $R$ and does not converge to 0 . For this one needs to verify by using the properties of the Greens function that $\left\|t^{\frac{1}{4}}\left(\partial_{x} \mathrm{e}^{t A} k\right)\right\|_{\infty}=\left\|\partial_{x} \mathrm{e}^{A} k\right\|_{\infty}$.

\subsection{Remark on non-uniqueness in dimension 4}

Example 4 becomes more interesting if one turns to higher dimension. Let $k(x)=\alpha_{d} \log (|x|)$ for $x \in \mathbf{R}^{d}$ with $\alpha_{d} \in \mathbf{R}$. As in Example $4 k \in \mathscr{B}$, but $k \notin \mathscr{Z}$. The function $k$ is not a weak solution for $d=2$ and all $\alpha_{d} \neq 0$. But as the weak derivative $\nabla k \in L_{\mathrm{loc}}^{2}$ in dimension $d \geq 3$, it is straightforward to check explicitly that both the weak formulation (7) and the mild formulation are satisfied with $\alpha_{d}=2-d$. 
A special case is dimension $d=4$, where $k$ is a stationary weak solution for any $\alpha_{d} \in \mathbf{R}$. But in that case the $\mathscr{B}$-norm of $k$ can be made arbitrarily small with $\alpha_{d} \rightarrow 0$, and thus by Theorem 1 and results in Section 3.6 below there is also a smooth mild solution starting at $k$.

In conclusion this example shows the non-uniqueness and lack of smoothness of mild solutions, at least in dimension $d=4$. Nevertheless there is no contradiction with our Theorem 1 , since this states uniqueness in $\mathscr{X}$ but $k \notin \mathscr{X}$, if considered as a function of time.

3.5 The fixed point argument

Define the map

$$
\mathscr{V}(h, k)(t)=-\int_{0}^{t} \Delta \mathrm{e}^{(t-s) A} \nabla h(s) \cdot \nabla k(s) d s=\int_{0}^{t} \mathrm{e}^{(t-s) A} B(h(s), k(s)) d s .
$$

Here we use the slightly modified concept of a mild solution. The main difference is that in contrast to Definition 2, we do not assume continuity in $t$. This needs to be established in an additional step.

Definition 3 We say that $h \in \mathscr{X}$ solves (9) with initial condition $h_{0} \in \mathscr{B}$, if for all $t>0$

$$
h(t)=\mathrm{e}^{t A} h_{0}+\mathscr{V}(h, h)(t) .
$$

We call $h \in \mathscr{X}_{R}$ a local mild solution, if (12) holds only on $t \in\left[0, R^{4}\right]$ for some $R>0$.

The following Lemma is crucial for the proof of uniqueness and existence. It verifies that the nonlinear part is locally Lipschitz continuous both in $\mathscr{X}$ and $\mathscr{X}_{R}$.

Lemma 6 The map $\mathscr{V}$ is bi-linear and continuous from $\mathscr{X} \times \mathscr{X}$ to $\mathscr{X}$ and from $\mathscr{X}_{R} \times \mathscr{X}_{R}$ to $\mathscr{X}_{R}$, for all $R>0$ such that

$$
\|\mathscr{V}(h, k)\|_{\mathscr{X}} \leq c_{4}\|k\|_{\mathscr{X}}\|h\|_{\mathscr{X}} \quad \text { and } \quad\|\mathscr{V}(h, k)\|_{\mathscr{X}_{R}} \leq c_{4}\|k\|_{\mathscr{X}_{R}}\|h\|_{\mathscr{X}_{R}} .
$$

Proof (Idea of proof) The bilinearity is obvious by the bilinearity of $B$. For the boundedness one uses $\|\nabla h \cdot \nabla k\|_{\infty} \leq t^{-1 / 2}\|k\|_{\mathscr{X}}\|h\|_{\mathscr{X}}$ together with an $L^{\infty}$ bound on $\Delta \mathrm{e}^{t A}$ given by an explicit calculation in terms of the Greens-function. For more details see [17, Lemma 4.2].

Using the previous Lemma, we can now state and prove our main result [17, Theorem 4.3] on global existence and local existence of unique solutions. Recall that $\left\|h_{0}\right\|_{\mathscr{B}_{R}} \rightarrow 0$ is true for $R \rightarrow 0$ for all $h_{0} \in \mathscr{Z}$.

Theorem 1 For any $d \geq 1$. Fix $0<\delta<1 / 4 c_{4}$, with $c_{4}$ from Lemma 6. If $\left\|h_{0}\right\|_{\mathscr{B}} \leq \delta$, then there exists a unique (global) solution in $\mathscr{X}$ of (9). Moreover, if $\left\|h_{0}\right\|_{\mathscr{B}_{R}} \leq \delta$, then there is a unique local solution in $\mathscr{X}_{R}$ of (9) on $\left[0, R^{4}\right]$. Finally, if $h_{0}$ is periodic and satisfies one of the bounds above, then the solution is also periodic. 
Proof The proof is based on a standard fixed point iteration argument. Choose $K$ such that

$$
1-\sqrt{1-4 c_{4} \delta} \leq 2 c_{4} K<1 .
$$

Define the iteration

$$
H_{0}=0, \quad H_{n+1}=\mathrm{e}^{t A} h_{0}+\mathscr{V}\left(H_{n}, H_{n}\right),
$$

then $\left\|H_{1}\right\|_{\mathscr{X}}=\left\|h_{0}\right\|_{\mathscr{B}} \leq \delta$. Now by Lemma $6\left\|H_{n+1}\right\| \mathscr{X} \leq \delta+c_{4}\left\|H_{n}\right\|_{\mathscr{X}}^{2}$. Thus by induction and the choice of $\delta$ and $K$, it follows that $\left\|H_{n}\right\|_{\mathscr{X}} \leq K$ for all $n$. Then

$$
\left\|H_{n+1}-H_{n}\right\|_{\mathscr{X}}=\left\|\mathscr{B}\left(H_{n}, H_{n}\right)-\mathscr{B}\left(H_{n-1}, H_{n-1}\right)\right\|_{\mathscr{X}} \leq 2 c_{4} K\left\|H_{n}-H_{n-1}\right\|_{\mathscr{X}}
$$

and so $\left(H_{n}\right)_{n \in \mathbf{N}}$ is convergent in $\mathscr{X}$ to a fixed point of (12).

The same proof works for local spaces, since all constants do not depend on $R$. The final statement of periodicity follows by translation invariance of (9) and uniqueness.

Remark 7 We remark without proof, that the solution of Theorem 1 is a mild solution in the sense of Definition 2 in the space $\mathscr{B}$. But the uniqueness is only verified in $\mathscr{X}$ or $\mathscr{X}_{R}$.

\subsection{Remark on smoothness of solutions}

In this section we state without proof the result that solutions granted by Theorem 1 are smooth in space and time for $t>0$. One approach is to follow the same methods of [48] and use for $m \geq 1$ the space

$$
\mathscr{X}^{m}=\left\{k: \mathbf{R}^{d} \rightarrow \mathbf{R}:\|k\|_{\mathscr{X}^{m}}:=\max _{0 \leq j \leq m}\|k\|_{\mathscr{X}, j}<\infty\right\}
$$

with the corresponding local version $\mathscr{X}_{R}^{m}$. Here the norm is

$$
\|k\|_{\mathscr{X}, m}:=\sup _{t>0}\left\{t^{\frac{m+1}{4}} \sum_{|\alpha|=m+1}\left\|D^{\alpha} k\right\|_{\infty}\right\}
$$

where $\|\cdot\|_{\mathscr{X}_{R}, m}$ is the corresponding local version, with $\alpha=\left(\alpha_{1}, \ldots, \alpha_{d}\right)$ and $D^{\alpha}=\partial_{x_{1}}^{\alpha_{1}} \ldots \partial_{x_{d}}^{\alpha_{d}}$ and $|\alpha|=\alpha_{1}+\cdots+\alpha_{d}$.

In order to go to higher regularity we repeat inductively the fixed-point argument in the space $\mathscr{X}^{m}$ or $\mathscr{X}_{R}^{m}$ to obtain the following proposition.

Proposition 4 (Proposition 6.3 of [17]) There exists $\delta>0$ such that if $\left\|h_{0}\right\|_{\mathscr{B}}<\delta$ (or $\left\|h_{0}\right\|_{\mathscr{B}_{R}}<\delta$ ), then the solution to (9) granted by Theorem 1 is in $\mathscr{X}^{m}$ (or $\mathscr{X}_{R}^{m}$ ) for all $m \geq 1$.

An immediate corollary is the following result on smoothness in space. Smoothness in time then follows from the PDE by a standard bootstrapping argument.

Corollary 1 Let $h$ be a solution of (9) in $\mathscr{X}_{R}$, with $0<R \leq \infty$. Then $h(t) \in C_{b}^{\infty}\left(\mathbf{R}^{d}\right)$ for all $t \in(0, R)$.

Remark 8 A more careful analysis would show that the solutions are analytic in space. We point out that Koch \& Lamm [66] have a more elegant proof of analyticity (in a different context). An advantage of the method used here is to provide the behaviour at $t=0$ of solutions through the spaces $\mathscr{X}^{m}$ and $\mathscr{X}_{R}^{m}$. 
3.7 Local existence for the stochastic problem

In this section we outline the basic idea of the proof of local existence for the stochastic PDE. Based on Section 2.4 we consider (8) instead of (9). The two additional terms are on one hand a linear term $B(Z, v)$ and a forcing term $B(Z, Z)$. Both are random due to the stochastic convolution $Z$, but for any fixed realization they are easily included in the deterministic fixed-point argument of Theorem 1 , provided $Z$ is sufficiently regular.

For instance, in the case of bounded intervals (i.e. $d=1$ ) with periodic boundary conditions and space-time white noise, we have that the stochastic convolution $Z$ and its derivative $\partial_{x} Z$ are continuous in both space and time. Compare to Proposition 1 for the spatial regularity and Remark 5 for time regularity.

Thus we can conclude that almost surely $\|Z\|_{\mathscr{X}_{R}} \rightarrow 0$ for $R \rightarrow 0$. Hence the equation can be uniquely solved in $\mathscr{X}_{R}$, for a random $R>0$.

An interesting question appears in the case of periodic boundary conditions and $d=2$, since for space-time white noise the convolution $Z$ just fails to be differentiable in space (see Proposition 3), and thus all results of this section are not applicable. In the next section we give details how to attack this problem.

Let us finally remark that for $d=2$ the stochastic convolution $Z$ will be differentiable if we consider slightly more regular noise. In that case all the machinery of this section again applies.

\section{Rough Noise - Not enough Regularity}

In this section we address the problem of proving the existence of local solutions for (1) in dimension $d=2$ where the regularity of solutions is too weak for the non-linear term. Fix for the whole section for simplicity of presentation $L=2 \pi$.

We follow an approach similar to the one used in [29] for a similar singular twodimensional problem with space-time white noise. Recently, there is a high interest in the analysis of non-linear PDEs that, like the one presented here, are forced by rough noise so that the non-linear term in principle is not well-defined. The meaning of the non-linearity is then recovered through probability. We refer to [52,54]. Two recent papers [50,53] have proposed general and powerful methods that would both apply to our equation (1) as well as to more difficult problems, where for instance the re-normalized infinite constant shows up in the equation. See also $[121,47,55]$.

The method we use here, based on Fourier expansion, works very well for our problem (1) and we believe it is, at least for this problem, neat and simple. It illustrates already the key problems one is facing.

The idea is to decompose the solution in a rough part having the low regularity dictated by the forcing and a remainder, slightly smoother. The non-linearity for the rough term is then defined as the limit of cut-offs via spectral Galerkin methods, thanks to the underlying Gaussian nature of the processes involved. This, roughly speaking, corresponds to a renormalization of the non-linearity, but here without any additional term, due to the fact that the Laplace in front of the gradient squared kills an infinite renormalization-constant, that would appear, as in KPZ [52], to compensate the divergence in the non-linearity.

The method works even for rougher noise at the price of a lower level of regularity. For even rougher noise the remainder fails to be regular enough, and then we need an additional term in the expansion of the solution, which would then lead to the method proposed in [50]. Here many terms are added in the expansion, and we discuss briefly on this in Section 4.4. 
Also the theory of regularity structures [53] uses a kind of Taylor expansion in terms of singular, noise dependent, terms and more regular parts, but this is on a level of greater generality.

\subsection{Setting and main result}

Here we use again the standard transformation $h=Z+v$ (cf. Section 2.4). We already saw that the stochastic convolution $Z$ is continuous in space and time, but it fails to have a derivative.

Since it turns out that $v$ is regular enough, the mixed product $B(v, Z)$ is well defined (cf. Lemma 2), by standard Sobolev theory, and we do not need to define this using the paracontrolled theory of [50], which allows to define the product for less regular functions. In the abstract theory of regularity structures these products are always defined.

The problems here originate only from the "squared distribution" $|\nabla Z|^{2}$ in $B(Z, Z)$. Once this term is properly defined as a limit of spectral approximations, for instance, we can work out a fixed point argument similar to [45] (see also [16] or Section 3).

In conclusion a mild solution $h$ of (1) is in this section a random process such that $v=h-Z$ is a solution of

$$
v(t)=S(t) h_{0}+\int_{0}^{t} S(t-s) B(v, v) d s+\int_{0}^{t} S(t-s)(\widetilde{B}(Z, Z)+2 B(Z, v)) d s
$$

where $\widetilde{B}(Z, Z)$ is the suitably modified product.

As in Section 3 we introduce time-weighted spaces, but now we use a Hilbert-space setting, in order to say more about norms of Fourier-series expansions of $B(Z, Z)$, which is not that easy in the space $\mathscr{X}$. Similar arguments are also possible in a Besov-space setting, but for simplicity of presentation, we restrict ourselves to the Hilbert-space setting only.

Given $\rho>0, \varepsilon>0$ and $T>0$, define

$$
\|u\|_{\varepsilon, T}:=\sup _{t \leq T} t^{\frac{\varepsilon}{4}}\|u(t)\|_{H^{1+\varepsilon}}
$$

We will find a solution of (13) by means of a fixed point argument for suitable $\rho$ and $T$ in the space

$$
\mathscr{X}(\varepsilon, \rho, T):=\left\{v \in C\left([0, T] ; \mathscr{L}^{2}\right):\|v\|_{\varepsilon, T} \leq \rho\right\} .
$$

Theorem 2 Let $h_{0} \in \mathscr{H}^{1}$ and $\varepsilon \in\left(0, \frac{1}{2}\right)$. There exist a stopping time $\tau_{h_{0}}$ and a solution $h$ of the mild formulation (cf. (13)) defined on $\left[0, \tau_{h_{0}}\right)$, such that $h \in C\left(\left[0, \tau_{h_{0}}\right) ; \mathscr{L}^{2}\right)$ and $h-Z \in C\left(\left(0, \tau_{h_{0}}\right) ; \mathscr{H}^{1+\varepsilon}\right)$. Moreover, $\mathbb{P}\left[\tau_{h_{0}}>0\right]=1$.

We will not give a detailed proof of this theorem, as it is in principle very similar to the proof in Section 3.5). Again the additional terms do not cause problems, provided they are sufficiently regular. For details see [18, Theorem 2.10]. For the mixed term we use Lemma 2 and the square of the Gaussian is defined in the next section.

Note that solutions are unique up to the minimum of both their stopping times in the space $\mathscr{X}(\varepsilon, \rho, T)$. Moreover, by standard methods one can continue uniquely the solution as continuous $\mathscr{H}^{1+\varepsilon}$-valued solutions, until they blow up.

Remark 9 With some effort one can show that $v$ is as regular as $\int_{0}^{t} S(t-s) \widetilde{B}(Z, Z) d s$. Here it is sufficiently regular to proceed with the fixed-point argument. If this is not the case, then additional expansions will be necessary (see Section 4.4). 
If this integral fails to be more regular than $Z$ alone, which might be the case for very rough noise or higher dimension, then there seems to be no way this method or any other like [50] or even regularity structures will work.

4.2 Additional regularity for powers of Gaussian

Here we discuss the key point in all the theory, which is the definition of the nonlinearity evaluated at Gaussian random variables that are not regular enough. The stochastic convolution $Z$ can be expanded in the complex Fourier basis (see (6)),

$$
Z(t)=\sum_{k \in \mathbf{Z}_{\star}^{2}} z_{k}(t) e_{k}, \quad z_{k}(t)=\int_{0}^{t} \mathrm{e}^{-|k|^{4}(t-s)} d \beta_{k}(s),
$$

where $\beta_{k}=\left\langle W(t), e_{k}\right\rangle, \beta_{-k}=\bar{\beta}_{k}$, and $\left(\beta_{k}\right)_{k \in \mathbf{Z}_{+}^{2}}$ is a sequence of independent complexvalued standard Brownian motions.

We saw in Lemma 3 that $Z$ is not sufficiently regular to define the non-linear term. But it turns out that, suitably defined, the term $B(Z, Z)$ does make sense.

If $u=\sum_{k \in \mathbf{Z}^{2}} u_{k} e_{k}$ and $v=\sum_{k \in \mathbf{Z}^{2}} v_{k} e_{k}$ are real valued, the non-linear term can be formally written in terms of the Fourier coefficients as

$$
B(u, v)=\sum_{k \in \mathbf{Z}_{\star}^{2}}|k|^{2}\left(\sum_{m+n=k} m \cdot n u_{m} v_{n}\right) e_{k} .
$$

Consider the stochastic convolution $Z$ and set for every $k \in \mathbf{Z}^{2}$,

$$
J_{k}(t)=\sum_{m+n=k} m \cdot n z_{m}(t) z_{n}(t)
$$

Formally, $B(Z, Z)=\sum_{k}|k|^{2} J_{k} e_{k}$, but Lemma 3 immediately tells us that

$$
J_{0}(t)=-\|\nabla Z(t)\|_{L^{2}}^{2}=\infty \quad \text { almost surely. }
$$

Likewise, we obtain, that the series in (14) is not absolutely convergent.

Following [29], we resum the terms $J_{k}(t)$ in a suitable way, in order to rely on cancellations, based on the fact that the $z_{m}$ are centred Gaussians. The term $J_{0}(t)$ should at first sight be the most problematic, since there is no hope to exploit any cancellation. But it is constant in space and thus cancelled by the Laplacian.

Given $N \geq 1$, let $H_{N}$ be the linear sub-space of $\mathscr{L}^{2}$ spanned by $\left(e_{k}\right)_{0<|k| \leq N}$. Let $\pi_{N}$ be the projection of $\mathscr{L}^{2}$ onto $H_{N}$ and define

$$
B_{N}(u, v)=B\left(\pi_{N} u, \pi_{N} v\right) .
$$

We define the operator $\widetilde{B}(Z, Z)$ as the limit of the sequence $\left(B_{N}(Z, Z)\right)_{N \geq 1}$.

Lemma 7 The approximation $\left(B_{N}(Z, Z)\right)_{N \geq 1} \gamma>0$. Thus the limit $\widetilde{B}(Z, Z)$ is almost-surely well-defined as an element of $\mathscr{H}^{-2-\gamma}$.

As the cancellation property used in this proof is essential for the whole theory, we repeat the proof of [18, Lemma 3.4]. 
Proof Let $J_{k}^{N}$ be the term analogous to $J_{k}$ for the projection $\pi_{N} Z$. If $N \leq N^{\prime}$, then

$$
\mathbb{E}\left[\left|J_{k}^{N}(t)-J_{k}^{N^{\prime}}(t)\right|^{2}\right]=\sum_{m_{1}+n_{1}=k m_{2}+n_{2}=k}^{N \leftrightarrow N^{\prime}} \sum_{1}^{N \leftrightarrow N^{\prime}} m_{1} \cdot n_{1} \cdot n_{2} \mathbb{E}\left[z_{m_{1}} z_{n_{1}} \bar{z}_{m_{2}} \bar{z}_{n_{2}}\right],
$$

where by the symbol $N \leftrightarrow N^{\prime}$ in the sum over $m, n$ we mean that the sum is extended only over those indices $m, n$ that satisfy $N<|m| \vee|n| \leq N^{\prime}$.

The sequence $\left(z_{m}\right)_{m \in \mathbf{Z}_{+}^{2}}$ is a family of independent centred Gaussian random variables. Moreover $\bar{z}_{m}=z_{-m}$. Using that odd moments of Gaussians are zero we can show that

$$
\mathbb{E}\left[z_{m_{1}} z_{n_{1}} \bar{z}_{m_{2}} \bar{z}_{n_{2}}\right] \neq 0 \quad\left\{\begin{array}{c}
\text { only if } m_{1}=m_{2} \text { and } n_{1}=n_{2} \\
\text { or if } m_{1}=n_{2} \text { and } m_{2}=n_{1}
\end{array}\right.
$$

Therefore,

$$
\begin{aligned}
& \mathbb{E}\left[\left|J_{k}^{N}(t)-J_{k}^{N^{\prime}}(t)\right|^{2}\right]=2 \sum_{m+n=k}^{N \leftrightarrow N^{\prime}}(m \cdot n)^{2} \mathbb{E}\left[\left|z_{m}\right|^{2}\left|z_{n}\right|^{2}\right] \leq \\
& \leq c \sum_{m+n=k}^{N \leftrightarrow N^{\prime}} \frac{(m \cdot n)^{2}}{|m|^{4}|n|^{4}}\left(1-\mathrm{e}^{-2|m|^{4} t}\right)\left(1-\mathrm{e}^{-2|n|^{4} t}\right) \leq c \sum_{m+n=k}^{N \leftrightarrow N^{\prime}} \frac{1}{|m|^{2}|n|^{2}} .
\end{aligned}
$$

The last series above can be estimated explicitly (see for example [19, Lemma 2.3] for details). Indeed since $|m| \vee|n| \geq N$,

$$
\sum_{m+n=k}^{N \leftrightarrow N^{\prime}} \frac{1}{|m|^{2}|n|^{2}} \leq \frac{2}{N^{\gamma}} \sum_{\substack{m+n=k \\|n| \leq|m|}}^{N \leftrightarrow N^{\prime}} \frac{1}{|m|^{2-\gamma}|n|^{2}} \leq \frac{2}{N^{\gamma}} \sum_{m+n=k}^{N \leftrightarrow N^{\prime}} \frac{1}{|m|^{2-\gamma}|n|^{2}} \leq \frac{c}{N \gamma|k|^{2-\gamma}}
$$

In conclusion,

$$
\mathbb{E}\left[\left\|\widetilde{B}_{N}(z, z)-\widetilde{B}_{N^{\prime}}(z, z)\right\|_{H^{-2-\gamma}}^{2}\right]=\sum_{k \in \mathbf{Z}_{\star}^{2}}|k|^{-2 \gamma} \mathbb{E}\left[\left|J_{k}^{N}-J_{k}^{N^{\prime}}\right|^{2}\right] \leq \frac{c}{N^{\gamma}} \sum_{k \in \mathbf{Z}_{\star}^{2}} \frac{1}{|k|^{2+\gamma}},
$$

and the term on the right hand side converges to zero as $N, N^{\prime} \rightarrow \infty$.

We shall need higher moments of $\widetilde{B}(z, z)$ for our considerations on the non-linear problem. We shall derive the claim from hyper-contractivity of Gaussian measures [82,105]. For Gaussian random variables it is well-known that all their moments are bounded by the second. By hyper-contractivity (Nelsons theorem) the same statements extends to polynomials of Gaussian random variables.

Proposition 5 Given $\gamma>0$ and $p>1$,

$$
\sup _{t>0} \mathbb{E}\left[\|\widetilde{B}(Z(t), Z(t))\|_{\mathscr{H}}^{p-2-\gamma}\right]<\infty
$$

From this result we obtain that $\widetilde{B}(Z, Z) \in L^{p}\left(0, T, \mathscr{H}^{-2-\gamma}\right)$ for all $p>1$. Higher regularity in time like continuity is possible, but not necessary for the fixed point argument. 
Proof For the second moment we can proceed as in the previous lemma,

$$
\mathbb{E}\left[\left|J_{k}^{N}\right|^{2}\right]=\sum_{\substack{m+n=k \\|m|,|n| \leq N}}(m \cdot n)^{2} \mathbb{E}\left[\left|z_{m}\right|^{2}\left|z_{n}\right|^{2}\right] \leq c \sum_{m+n=k} \frac{1}{|m|^{2}|n|^{2}} \leq \frac{c}{|k|^{2}} \log (1+|k|) .
$$

And thus

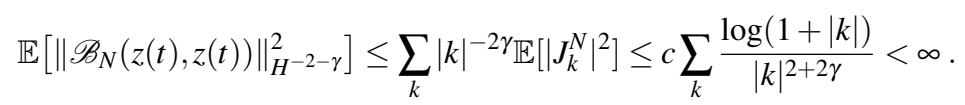

To prove that all higher moments are finite, as outlined before we can rely on Theorem I.22 of [105]. We skip the proof here.

\subsection{Other regularizations}

Here we consider one regularizing method different from the spectral Galerkin method used to define $\widetilde{B}(Z, Z)$. In principle all approximations may lead to a different renormalization $\widetilde{B}$, but it turns out that in numerous cases they are all the same, and thus the solution of the renormalized problem (13) is always the same. For an abstract result of different regularizations see [18, Thm. $2.3 \& 2.4]$.

Here we only give one example of such regularizations using a convolution operator. Consider a regularizing operator $\Phi_{N}$ acting on the process $Z$, or equivalently on $W$, as the convolution commutes with the semigroup. In the previous section we discussed the case $\Phi_{N}=\pi_{N}$ of spectral Galerkin projections.

Example 5 (Convolution operator) Given a non-negative smooth function $q$ with support contained in a small neighbourhood of the origin and such that $\int q(x) d x=1$, let $q_{N}$ be the $2 \pi$-periodic extension on the flat torus $[0,2 \pi]^{2}$ of $z \mapsto q(N z)$. Define

$$
\Phi_{N} f(x)=\int_{[0,2 \pi]^{2}} N^{2} q_{N}(x-y) f(y) d y
$$

The operators $\Phi_{N}$ are self-adjoint and diagonal in the Fourier basis. Denote by $\phi_{k}^{N}$ the eigenvalues of $\Phi_{N}$. These are (up to constant) given by the Fourier coefficients of $z \mapsto N^{2} q(N z)$.

We need some decay condition, in order for $\Phi_{N}$ to have a regularizing effect that is strong enough. Here is is sufficient that $\left|\phi_{k}^{N}\right| \lesssim|k|^{-\eta}$ for some $\eta>0$, which is assured by $q \in H^{\eta}$ for some $\eta>0$.

In order to have the regularization disappear in the limit, we need $\phi_{k}^{N} \rightarrow 1$ as $N \rightarrow \infty$. This can be checked using the fact that $q$ is supported around 0 ,

$$
q_{k}^{N}=N^{2} \int_{[-\pi, \pi]^{2}} q_{N}(x) e_{-k}(x) d x=\int_{[-\pi, \pi]^{2}} q(z) e_{-k / N}(z) d z \longrightarrow 1 \quad \text { for } N \rightarrow \infty .
$$

\subsection{Rougher noise}

As it is apparent by the previous sections, space-time white noise is the borderline case between the standard theory for mild solutions in Section 3.7 and the additional work presented in Theorem 2. 
Nevertheless, it is possible to consider rougher noise than space-time. In view of the computations needed to define $\widetilde{B}(Z, Z)$ (Lemma 7) it is reasonable to consider a simplified case, namely when the covariance operator of the driving Wiener process is diagonal in the Fourier basis. This is the somewhat opposite case of Example 5, when we consider kernels $q$ given by a distribution instead of a function.

Consider a bounded linear operator $\Phi$ on $\mathscr{L}^{2}$ and assume for the rest of this section the following properties,

- $\Phi \mathrm{e}_{k}=\phi_{k} e_{k}$ for every $k \in \mathbf{Z}_{\star}^{2}$,

- there is $\beta>0$ such that $\left|\phi_{k}\right|^{2} \leq c|k|^{\beta}$ for every $k \in \mathbf{Z}_{\star}^{2}$.

The value $\beta=0$ corresponds to noise probably as bad as space-time white noise. Moreover, the definition of $\widetilde{B}(Z, Z)$ imposes a structural restriction that limits the range of possible values of $\beta$ to $\beta<1$. The main problem of $\beta>1$ is that $\mathbb{E}\left[\left|J_{k}(t)\right|^{2}\right]$ might no longer converge, and our simple renormalization is not good enough to handle this case. Additional renormalization seems to be necessary.

Consider now the SPDE with rougher noise

$$
d h=(A h+B(h, h)) d t+d \Phi W,
$$

and the OU-process

$$
Z(t)=\int_{0}^{t} S(t-s) \Phi d W(s) .
$$

The same ideas presented earlier in Section 4, when slightly modified to take into account the parameter $\beta$, lead to the following result. See [18, Theorem 2.9].

Theorem 3 Assume $\beta \in\left(0, \frac{2}{3}\right)$ and let $h_{0} \in \mathscr{H}^{1}$ and $\varepsilon \in\left(\frac{\beta}{2},(1-\beta) \wedge \frac{1}{2}\right)$.

Given the covariance operator $\Phi$, there exist a stopping time $\tau_{h_{0}}$ and a solution $h$ of (15) understood as $h=v+Z$ with $Z$ from (16), where $v$ satisfies the mild formulation (13) on $\left[0, \tau_{h_{0}}\right)$. Moreover, $h \in C\left(\left[0, \tau_{h_{0}}\right) ; \mathscr{L}^{2}\right), h-z \in C\left(\left(0, \tau_{h_{0}}\right) ; \mathscr{H}^{1+\varepsilon}\right)$, and $\mathbb{P}\left[\tau_{h_{0}}>0\right]=1$. Finally, two such solutions are unique up to the minimum of their stopping time.

It is not obvious, but the restriction $\beta<\frac{2}{3}$ appears due to the term $B(v, Z)$ in the mild formulation (13). When the noise is too rough, the auxiliary function $v$ is not enough regular to ensure that the product $B(v, Z)$ is well-defined. We could either use the paracontrolled approach in [50] in order to define the product of two not sufficiently regular functions, or immediately go to higher terms in the expansions.

To overcome the difficulty for $\beta \in\left[\frac{2}{3}, 1\right)$ in the decomposition of $h$, namely $h=u+\zeta+$ $Z$, where

$$
\zeta(t)=\int_{0}^{t} S(t-s) \widetilde{B}(Z(s), Z(s)) d s,
$$

and from (13) we see that $u=v-\zeta$ is a solution of the mild formulation

$$
u(t)=S(t) h_{0}+\int_{0}^{t} S(t-s)[B(u, u)+2 B(u, Z)+2 B(u, \zeta)+2 \widetilde{B}(\zeta, Z)+\widetilde{B}(\zeta, \zeta)] d s .
$$

We now hope that $u$ is sufficiently regular that $B(u, Z)$ and also $B(u, \zeta)$ are defined.

Moreover, we need to suitably define the products $\widetilde{B}(\zeta, z)$ and $\mathscr{B}(\zeta, \zeta)$. We can proceed as we did for $\widetilde{B}(z, z)$ in Section 4.2 , by exploiting the cancellations in the expectations of these processes. Even if we would have enough regularity, in order to define the products directly, these Gaussian cancellations yield better regularity of the products.

We are able then to state the following result from [18, Theorem 2.10]. 
Theorem 4 Assume $\beta \in(0,1)$ and let $h_{0} \in \mathscr{H}^{1}$ and $\varepsilon \in\left(\frac{\beta}{2}, \frac{1}{2}\right)$. Given the covariance operator $\Phi$, there exist a stopping time $\tau_{h_{0}}$ and a solution $h$ of (15) understood as $h=u+\zeta+Z$, where $Z$ is given by (16), $\zeta$ by (17) and $u=h-z-\zeta$ satisfies the mild formulation (18) on $\left[0, \tau_{h_{0}}\right)$. Moreover, $h \in C\left(\left[0, \tau_{h_{0}}\right) ; \mathscr{L}^{2}\right), u \in C\left(\left(0, \tau_{h_{0}}\right) ; \mathscr{H}^{1+\varepsilon}\right)$ and $\mathbb{P}\left[\tau_{h_{0}}>0\right]=1$. Finally, two such solutions are unique up to the minimum of their stopping time.

In principle, if a suitable regularization of $\widetilde{B}$ would allow for even less regular noise with higher values of $\beta$, we could go to even higher terms in the expansion.

\section{Global existence}

In this section we first focus on the one-dimensional case on the interval $[0, L]$, as only here we have useful uniform global a-priori estimates for the spectral Galerkin-approximation. See (3). We comment on the case $d=2$ in detail later in this section.

There are two standard ways of treating the global existence of solutions. The first one relies on the fixed point argument, which was explained in Section 3. Here the result is that small initial data yields global existence for all times. But in general one can only show that local solutions are smooth and continue until a possible blow up, which we discuss later in Section 6.

The other approach relies on an approximation like the spectral Galerkin method, for example, and uses the following steps:

- Energy type / a-priori estimates for the approximation, like (3) for instance.

- Compactness of the approximating sequence (by using uniform bounds) yields a convergent subsequence

- Passage to the limit in the approximation. The key problem here is usually the continuity of the nonlinear term in the weak topology provided by the compactness result.

This program was carried out for many models in numerous publications. For our model and $d=1$ it was implemented by [108], and for the stochastically perturbed equation in [11, $13,9]$. See also [74,91]. In all cases global solutions are only bounded uniformly in $\mathscr{L}^{2}$, which is not enough to ensure uniqueness provided for instance by the fixed-point argument.

A serious difficulty arises for SPDEs, if one only repeats path-wise the deterministic result, as the choice of the subsequence will depend on the realization under consideration, and thus in general the limit will no longer be a measurable random variable. This only works with stochastic equations that admit a strong (in terms of probability) solution. On the other hand we may consider a weaker (again in terms of probability) notion of solution, characterized only by the law. In this case for the approximations we consider compactness of the corresponding probability measures (Prokhorov's theorem), to obtain a subsequence independent of the realizations. The price one needs to pay is that the convergence is too weak to pass to the limit in the nonlinearity. But one can rely on the Skorokhod representation theorem in order to obtain, on a different probability space and with different input data (with the same distributions though), an almost surely convergent subsequence, which is sufficient for the passage to the limit. This procedure only yields a so called Martingale solution. See for instance [32,40].

Here we we intend to solve the equation for all initial conditions on the same probability space and follow the results of [9] that use the method developed by Flandoli \& Romito [42] and [44] in order to establish the existence of weak solutions having the Markov property. For the precise formulation of the concept of solution see Definitions 5 and 6 below. 
The method is essentially based on showing a multi-valued version of the Markov property for sets of all possible solutions and then applying a clever selection principle (Theorem 5). The original idea is due to Krylov [67] (see also Stroock \& Varadhan [109, Chapter 12]).

A key point in this analysis is the definition of weak martingale solutions. The selection procedure needs to handle solutions which incorporate all the necessary bounds on the solution of the SPDE. These bounds must be compatible with the underlying Markov structure.

\subsection{Setting}

Let $\Omega=C\left([0, \infty) ; \mathscr{H}^{-4}\right)$ and let $\mathscr{B}$ be the $\sigma$-algebra of Borel subsets of $\Omega$. Let $\xi:[0, \infty) \times$ $\Omega \rightarrow \mathscr{H}^{-4}$ be the canonical process on $\Omega$, defined as $\xi(t, \omega)=\omega(t)$.

For each $t \geq 0$, let $\mathscr{B}_{t}=\sigma[\xi(s): 0 \leq s \leq t]$ be the $\sigma$-field of events up to time $t$ and $\mathscr{B}^{t}=\sigma[\xi(s): s \geq t]$ be the $\sigma$-field of events after time $t$. The $\sigma$-field $\mathscr{B}_{t}$ can be seen as the Borel $\sigma$-field of $\Omega_{t}=C\left([0, t] ; \mathscr{H}^{-4}\right)$ and, similarly, $\mathscr{B}^{t}$ as the Borel $\sigma$-field of $\Omega^{t}=C\left([t, \infty] ; \mathscr{H}^{-4}\right)$. Notice that both $\Omega_{t}$ and $\Omega^{t}$ can be seen as Borel subsets of $\Omega$ (by restriction to corresponding sub-intervals). Define finally the forward shift $\Phi_{t}: \Omega \rightarrow \Omega^{t}$, defined as

$$
\Phi_{t}(\omega)(s)=\omega(s-t), \quad s \geq t .
$$

Given a probability measure $P$ on $(\Omega, \mathscr{B})$ and $t>0$, we shall denote by $\left.\omega \mapsto P\right|_{\mathscr{B}_{t}} ^{\omega}$ : $\Omega \rightarrow \Omega^{t}$ a regular conditional probability distribution of $P$ given $\mathscr{B}_{t}$. Notice that $\Omega$ is a Polish space and $\mathscr{B}_{t}$ is countably generated, so a regular conditional probability distribution does exist and is unique, up to $P$-null sets.

In particular, $\left.P\right|_{\mathscr{B}_{t}} ^{\omega}\left[\omega^{\prime}: \xi\left(t, \omega^{\prime}\right)=\omega(t)\right]=1$ and, if $A \in \mathscr{B}_{t}$ and $B \in \mathscr{B}^{t}$, then

$$
P[A \cap B]=\left.\int_{A} P\right|_{\mathscr{B}_{t}} ^{\omega}[B] P(d \omega) .
$$

One can see the probability measures $\left(\left.P\right|_{\mathscr{B}_{t}} ^{\omega}\right)_{\omega \in \Omega}$ as measures on $\Omega$ such that $\left.P\right|_{\mathscr{B}_{t}} ^{\omega}\left[\omega^{\prime} \in\right.$ $\Omega: \omega^{\prime}(s)=\omega(s)$, for all $\left.s \in[0, t]\right]=1$ for all $\omega$ in a $\mathscr{B}_{t}$-measurable $P$-full set. We finally define the reconstruction of probability measures (details on this can be found in Stroock \& Varadhan [109, Chapter 6]).

Definition 4 Given a probability measure $P$ on $(\Omega, \mathscr{B}), t>0$ and a $\mathscr{B}_{t}$-measurable map $Q: \Omega \rightarrow \operatorname{Pr}\left(\Omega^{t}\right)$ such that $Q_{\omega}\left[\xi_{t}=\omega(t)\right]=1$ for all $\omega \in \Omega, P \otimes_{t} Q$ is the unique probability measure on $(\Omega, \mathscr{B})$ such that

1. $P \otimes_{t} Q$ agrees with $P$ on $\mathscr{B}_{t}$,

2. $\left(Q_{\omega}\right)_{\omega \in \Omega}$ is a regular conditional probability distribution of $P \otimes_{t} Q$, given $\mathscr{B}_{t}$.

For shorthand notation denote by $\operatorname{Pr}(X)$ the set of all probability measures on the space $X$ equipped with the Borel- $\sigma$-algebra.

5.2 The martingale problem

We state the weak formulation of (1) in terms of measures.

Definition 5 (weak martingale solution) Given $\mu_{0} \in \operatorname{Pr}\left(\mathscr{L}^{2}\right)$, a probability measure $P$ on $(\Omega, \mathscr{B})$ is a solution, starting at $\mu_{0}$, to the martingale problem associated to equation (1) if 
[W1] $P\left[L_{l o c}^{2}\left([0, \infty) ; \mathscr{H}^{1}\right)\right]=1$,

[W2] for every $\varphi \in \mathscr{D}^{\infty}$, the process $\left(M_{t}^{\varphi}, \mathscr{B}_{t}, P\right)_{t \geq 0}$, defined $P$-a. s. on $(\Omega, \mathscr{B})$ as

$$
M_{t}^{\varphi}=\langle\xi(t)-\xi(0), \varphi\rangle_{\mathscr{L}^{2}}+\int_{0}^{t}\left\langle\xi(s), \partial_{x}^{4} \varphi\right\rangle_{\mathscr{L}^{2}} d s+\int_{0}^{t}\left\langle\left(\partial_{x} \xi(s)\right)^{2}, \partial_{x}^{2} \varphi\right\rangle_{\mathscr{L}^{2}} d s
$$

is a Brownian motion with variance $t\|\varphi\|_{\mathscr{L}^{2}}^{2}$,

[W3] the marginal at time 0 of $P$ is $\mu_{0}$

Remark 10 It is not difficult to prove that the definition of weak martingale solution given above coincides with the usual definition given in terms of existence of an underlying probability space and a Wiener process. This equivalence is proved in Flandoli [40] for the NavierStokes equations and one can proceed similarly in this case, but we do not focus on this here.

Given all the $M_{t}^{\varphi}$ one can rely on the Kolmogorov extension theorem, in order to show that there is a Wiener-process $W$ such that $M_{t}^{\varphi}=\langle W(t), \varphi\rangle$, but here we can proceed in a simpler way, and define $W$ directly. Define, for every $k \in \mathbf{N}$, the process $\beta_{k}(t)=M_{t}^{e_{k}}$. Under any weak martingale solution $P$, the $\left(\beta_{k}\right)_{k \in \mathbf{N}}$ are a sequence of independent one-dimensional standard Brownian motions. By definition, one only needs to show the independence, which is also immediate. Let us only show that they are uncorrelated and neglect all other technical difficulties. By Definition 5

$$
2 \mathbb{E}\left[\beta_{k}(t) \beta_{\ell}(t)\right]=2 \mathbb{E}\left[M_{t}^{e_{k}} M_{t}^{e_{\ell}}\right]=\mathbb{E}\left[M_{t}^{e_{k}+e_{\ell}}\right]^{2}-\mathbb{E}\left[M_{t}^{e_{k}}\right]^{2}-\mathbb{E}\left[M_{t}^{e_{\ell}}\right]^{2}=0 .
$$

Define now the Wiener process $W$ by (5) and the Ornstein-Uhlenbeck process $Z$ by (6), both as random variables on $\Omega$. Recall that the process $Z$ can be defined path-wise, without relying on Itō-calculus. In the following lemma we summarise all the regularity result for $Z$ that we shall use for our result here. See also Section 2.1, where these properties are partly derived.

Lemma 8 Given a weak martingale solution $P$, let $Z$ be the corresponding OU-process defined in (6). Then

1. For every $p \geq 1$ and $T>0, Z \in L^{p}\left(\Omega \times(0, T) ; \mathscr{W}^{1,4}\right)$. Moreover there is $\lambda>0$,

$$
\sup _{T>0} \frac{1}{T} \mathbb{E}^{P}\left[\int_{0}^{T} \exp \left\{\lambda|Z(t)|_{\mathscr{W} 1,4}^{2}\right\} d t\right]<\infty .
$$

2. For every $p \geq 1$ and $\gamma \in\left[0, \frac{3}{2}\right), Z \in L^{p}\left(\Omega ; L_{\text {loc }}^{\infty}\left([0, \infty), \mathscr{H}^{\gamma}\right)\right)$.

3. $Z$ is $P-a$. s. weakly continuous with values in $\mathscr{H}^{\gamma}$, for every $\gamma \in\left[0, \frac{3}{2}\right)$.

Note that we carefully need to check that these properties are really true for any weak martingale solution $P$. We are not allowed to use versions of the process $Z$. Thus continuity in time is not a property we can rely on.

Definition 6 (energy martingale solution) Given $\mu_{0} \in \operatorname{Pr}\left(\mathscr{L}^{2}\right)$, a probability measure $P$ on $(\Omega, \mathscr{B})$ is an energy martingale solution to equation (1) starting at $\mu_{0}$ if

[E1] $P$ is a weak martingale solution starting at $\mu_{0}$,

[E2] $P\left[V \in L_{l o c}^{\infty}\left([0, \infty) ; \mathscr{L}^{2}\right) \cap L_{l o c}^{2}\left([0, \infty) ; \mathscr{H}^{2}\right)\right]=1$,

[E3] there is a set $T_{P} \subset(0, \infty)$ of null Lebesgue measure such that

$$
\text { for all } s \notin T_{P} \text { and all } t \geq s, \quad P\left[\mathscr{E}_{t}(V, Z) \leq \mathscr{E}_{s}(V, Z)\right]=1,
$$


where we used the standard transformation $V(t, \omega)=\xi(t, \omega)-Z(t, \omega)$, for $t \geq 0$, and the energy functional $\mathscr{E}$ is defined as

$$
\mathscr{E}_{t}(v, z)=\frac{1}{2}\|v(t)\|_{\mathscr{L}^{2}}^{2}+\int_{0}^{t}\left(\|v\|_{\mathscr{H}^{2}}^{2}-\left\langle 2 \partial_{x} v \cdot \partial_{x} z+\left(\partial_{x} z\right)^{2}, \partial_{x}^{2} v\right\rangle_{\mathscr{L}^{2}}\right) d s
$$

for $v \in L^{\infty}\left([0, t] ; \mathscr{L}^{2}\right) \cap L^{2}\left([0, t] ; \mathscr{H}^{2}\right)$ and $z \in L^{4}\left([0, t] ; \mathscr{W}^{1,4}\right) \cap L^{\infty}\left([0, t] ; \mathscr{H}^{1}\right)$.

Remark 11 (The equation for $V$ ) Let $P$ be an energy martingale solution, then it is easy to see (cf. Section 2.4) that $V$ is a weak solution to the equation,

$$
\partial_{t} V+\partial_{x}^{4} V=-\partial_{x}^{2}\left[\left(\partial_{x} V+\partial_{x} Z\right)^{2}\right], \quad V(0)=\xi(0) .
$$

Remark 12 (Finiteness of the energy) Given an energy martingale solution $P$, then the energy $\mathscr{E}_{t}$ is almost surely finite under $P$. Indeed, by [E2], it follows that $V(t)$ is $P$-a. s. weakly continuous in $\mathscr{L}^{2}$ (see for example Lemma 3.1.4 of Temam [114]), and so the function $t \mapsto\|V(t)\|_{\mathscr{L}^{2}}^{2}$ is defined point-wise in the energy estimate. Similarly, the other terms are also $P$-a. s. finite by [E2] and the regularity properties of $Z$ under $P$.

Remark 13 We remark that property [E3] is quite strong and that the energy inequality is an intrinsic regularity property of the solution to the original problem (1), and does not depend on the splitting $V+Z$. Similar estimates follow from [E3] for any other transformation $\xi=$ $V+Z_{\alpha}$, where $Z_{\alpha}(t)$ solves $d Z_{\alpha}=-\left[\partial_{x}^{4} Z-\alpha Z_{\alpha}\right] d t+d W$. A similar result was discussed in [93] for the Navier-Stokes equations.

\subsection{Existence of Markov solutions}

This section is devoted to the existence of Markov solutions for equation (1). To such aim, define for each $f \in \mathscr{L}^{2}$,

$$
\mathscr{C}(f)=\left\{P: P \text { is an energy martingale solution starting at } \boldsymbol{\delta}_{f}\right\} .
$$

We state the main theorem of this section on existence of global solutions.

Theorem 5 There exists a family $\left(P_{f}\right)_{f \in \mathscr{L}^{2}}$ of probability measures on $(\Omega, \mathscr{B})$ such that for each $f \in \mathscr{L}^{2}, P_{f}$ is an energy martingale solution with initial distribution $\delta_{f}$, and the a. $s$. Markov property holds: there is a set $T_{P} \subset(0, \infty)$ with null Lebesgue measure such that for all $s \notin T_{P}$, all $t \geq s$ and all bounded measurable $\phi: \mathscr{L}^{2} \rightarrow \mathbf{R}$,

$$
\mathbb{E}^{P}\left[\phi\left(\xi_{t}\right) \mid \mathscr{B}_{t}\right]=\mathbb{E}^{P_{\xi_{s}}}\left[\phi\left(\xi_{t-s}\right)\right] .
$$

Once the existence of at least one Markov family of solutions is ensured, the analysis of such solutions goes further. Indeed, the selection principle provides a family of solutions whose dependence with respect to the initial conditions is just measurability. By slightly restricting the set of initial condition, this dependence can be improved to continuity in the total variation norm (or strong Feller in terms of the corresponding transition semigroup). We do not study this in detail here, but this is crucial, if we want to study long term dynamics in terms of invariant measures.

Proof (Idea of proof) We follow [9, Theorem 3.1]. It is sufficient to show that the family $(\mathscr{C}(x))_{x \in \mathscr{L}^{2}}$ defined above is an almost sure pre-Markov family. We recall now the various properties of an a. s. pre-Markov family, which we need to verify in order to prove the theorem (see also Definition 2.5 of Flandoli \& Romito [44]). 
1. Each $\mathscr{C}(x)$ is non-empty, compact and convex, and the map $x \rightarrow \mathscr{C}(x)$ is measurable with respect to the Borel $\sigma$-fields of the space of compact subsets of $\operatorname{Pr}(\Omega)$ (endowed with the Hausdorff measure).

2. For each $x \in \mathscr{L}^{2}$ and all $P \in \mathscr{C}(x), P\left[C\left([0, \infty) ; \mathscr{L}_{\text {weak }}^{2}\right)\right]=1$, where $\mathscr{L}_{\text {weak }}^{2}$ is the space $\mathscr{L}^{2}$ with the weak topology.

3. For each $x \in \mathscr{L}^{2}$ and $P \in \mathscr{C}(x)$ there is a set $T \subset(0, \infty)$ with null Lebesgue measure, such that for all $t \notin T$ the following properties hold:

(a) (disintegration) there exists $N \in \mathscr{B}_{t}$ with $P(N)=0$ such that for all $\omega \notin N$

$$
\omega(t) \in \mathscr{L}^{2} \quad \text { and }\left.\quad P\right|_{\mathscr{B}_{t}} ^{\omega} \in \Phi_{t} \mathscr{C}(\omega(t)) \text {; }
$$

(b) (reconstruction) for each $\mathscr{B}_{t}$-measurable map $\omega \mapsto Q_{\omega}: \Omega \rightarrow \operatorname{Pr}\left(\Omega^{t}\right)$ such that there is $N \in \mathscr{B}_{t}$ with $P(N)=0$ and for all $\omega \notin N$

we have that $P \otimes_{t} Q \in \mathscr{C}(x)$.

$$
\omega(t) \in \mathscr{L}^{2} \quad \text { and } \quad Q_{\omega} \in \Phi_{t} \mathscr{C}(\omega(t)),
$$

The validity of these properties is verified [9]. Property (1) is verified by spectral Galerkin methods and compactness of probability measures (see [11]). Property (2) is a weak regularity property, while the main work is in property (3). From the almost sure pre-Markov family using the selection principle of Krylov [67] (see also Stroock \& Varadhan [109, Chapter 12]) the existence of an almost sure Markov process follows.

\subsection{Remarks on invariant measures}

Let us discuss the long time behaviour of the model. We are able to show that any Markov solution has a unique invariant measure whose support covers the whole state space. In principle the existence of stationary states has been already proved by Blömker and Hairer [13] using spectral Galerkin approximation. But this is not useful in this framework, as we have a transition semigroup that depends on the generic selection under analysis, which is in general not obtained by a suitable limit of Galerkin approximations.

We finally remark that, even though our results show that every Markov solution is strong Feller and converges to its own invariant measure, well posedness is still an open problem for this model and these result essentially do not improve our knowledge on the problem. Even the invariant measures are different, as they depend from different Markov semigroups.

Let us remark that provided one can show that all possible invariant measures for all Markov-selections are the same, then the energy martingale solution is unique, see [92]. See also $[38,106]$ for some results loosely in the same direction.

\subsection{A comparison with previous results on the Markov property}

There are several mathematical interests in this model, in comparison with the theory developed in Flandoli \& Romito $[42,44]$ for the Navier-Stokes equations. Essentially, in this model we have been able to find the natural space for the Markov dynamics, thus showing the existence of the (unique) invariant measure. The corresponding result for Markov selections of the Navier-Stokes equations with noise can be found in [92], see also [98,95].

Another challenge of this model has concerned the analysis of the energy inequality. Here the physics of the model requires a noise white in time and space, while the analysis developed in the above cited papers has been based on a trace-class noise with quite regular trajectories. 
Finally, we remark that there is a different approach to handle the existence of solutions with the Markov property, based on spectral Galerkin methods, which has been developed by Da Prato and Debussche [30] (see also Debussche \& Odasso [35]) for the Navier-Stokes equations. These techniques are not yet applied for the model analysed here.

\subsection{Remarks about 2D}

In order to complete the same program for the (physically relevant) two-dimensional case $d=2$, which is still open, there are several problems that need to be faced.

- When dealing with space-time white noise it turns out that the expected smoothness of the solution is not enough to define the non-linear term. This is discussed in Section 4 . Here we would need to take into account that we need to define $\widetilde{B}$ for any given Martingale-solution, but the equation defining the Martingale-solution already contains $\widetilde{B}$. But with slightly more regular $Z$ or $W$ this whole problem does not appear.

- In contrast with the one-dimensional case, existence of global weak solutions is harder, due to the lack of reasonable energy estimates in $\mathscr{L}^{2}$. Existence of weak solutions without noise has been proved in [120], using a-priori bounds derived from the estimate of $\int \mathrm{e}^{h} d x$, which cannot be used for any approximation by Galerkin methods. They rely on Roethe's method, a fully implicit discretization of the time derivative.

- Our proof of continuity of the laws in total variation $[44,43,9]$ relies on the weakstrong uniqueness principle, namely uniqueness of local solutions in the class of weak solutions. The principle fails in two dimensions, and this a serious obstacle for the proof of unique ergodicity.

\section{Blow-Up Criteria and Regularity}

In this section we focus on the deterministic equation in dimension $d=1$ and study possible singularities and a possible blow up, which existence is still wide open. These are usually standard results for PDEs of this type, and some are well known for instance for 3D-Navier Stokes.

Based on energy-type estimates, we state standard Leray type estimates for lower bounds on blow up in terms of $\mathscr{H}^{\alpha}$-norms. Moreover, we give a few examples for the fact that a blow up would lead to blow up of all critical norms. Finally, we state a well known result for an upper bound on the Hausdorff-dimension of the set of singularities in time.

All results for regularity and Leray type estimates are based on energy estimates. Many are optimal in the sense that they also hold for complex valued solutions. We will see in Section 7 that some complex valued solutions will blow up in finite time.

\subsection{Blow up profile}

In this section we discuss some properties of a possible blow up. First, from the concept of critical scaling one expects that all norms with higher regularity than the critical norm will also blow up, in particular all $\mathscr{H}^{s}$-norms with $s>1 / 2$ should blow up.

Let us first give examples of blow-up profiles $v=h(\tau)$ possible at the blow-up time $\tau$. For simplicity of presentation we only discuss $\mathscr{H}^{s}$-norms, as they are easier to compute. But one should also look at the spaces $\mathscr{B}$ or $\mathscr{Z}$ from Section 3. 
- If $v$ exhibits a jump like $\operatorname{sign}(x)$, then the Fourier-coefficients decay like $1 / k$, and thus $v$ is in $H^{s}$ if and only if $s<\frac{1}{2}$.

- If $v$ exhibits a logarithmic pole like $\log (|x|)$, then the Fourier-coefficients decay like $1 / k$, and thus $v$ is in $H^{s}$ if and only if $s<\frac{1}{2}$. See also Example 4.

- If $v$ exhibits a cusp like $|x|^{\alpha}$ for $\alpha \in(0,1)$, then the Fourier-coefficients decay like $|k|^{-(1+\alpha)}$, and thus $v$ is in $H^{1 / 2+\varepsilon}$, and not a possible blow up. It admits a unique solution that would continue the solution.

Remark 14 (Stationary solutions) The $\mathscr{L}^{2}$ estimates (3) show that the only possible stationary solution is $h \equiv 0$, as for global smooth solutions $\|h(t)\|_{\mathscr{L}^{2}} \rightarrow 0$ for $t \uparrow \infty$. On the other hand the problem is one-dimensional, so we can try to solve the equation for stationary solutions $\partial_{x}^{4} h+\partial_{x}^{2}\left(\left|\partial_{x} h\right|^{2}\right)=0$, explicitly. By direct computations, we get all solutions

$$
\begin{aligned}
& h(x)=c_{1}+\log \left|1+c_{2} x\right|, \\
& h(x)=c_{1}+\log \left|\cosh b x+\frac{c_{2}}{b} \sinh b x\right|, \\
& h(x)=c_{1}+\log \left|b \cos b x+c_{2} \sin b x\right| .
\end{aligned}
$$

We remark that the singularities are log-like profile, and some of them also satisfy periodic boundary conditions. But none of them is sufficiently regular, to be a mild or weak solution. Nevertheless, they are exactly at the borderline of regularity, where the local uniqueness results of Section 3 fail. See also Example 4.

\subsection{Regularity Criteria}

In principle the following should be true:

Meta-theorem. If a solution is bounded in a critical space, then it is unique, and does not have a blow up. This means that the unique local solution exists as long as at least one (hence all, as the solution is then proved to be regular) of the critical norms is finite over the time horizon.

The main obstruction to the application of the meta-theorem is the boundedness of critical quantities. While there are cases (the Navier-Stokes equations in dimension two, for instance) where the a-priori estimates provide such bounds, this does not happen, as far as we know, in the problem studied here.

For simplicity, in the rest of the section we focus only on examples in a simple setting and consider solutions with sufficiently smooth initial condition, in order to have simple energy type estimates for the $\mathscr{H}^{s}$-norms without any trouble at $t=0$. The standing assumption of this section is:

Assumption 6 Let $h_{0} \in C^{\infty}$ and $h=h\left(\cdot, h_{0}\right)$ be the unique local solution of the deterministic equation with $d=1$ started at $h_{0}$. Furthermore let $\tau\left(h_{0}\right)$ be the maximal time of $h$, and suppose $\tau\left(h_{0}\right)<\infty$ implies $\lim \sup _{t \rightarrow \tau}\|h(t)\|_{\mathscr{H} s}=\infty$ for one s. Moreover, by regularity $h$ is $C^{\infty}$ in space and time for $t \in\left[0, \tau\left(h_{0}\right)\right)$.

Theorem 7 Under Assumption 6 with $s=1$, i.e. the subcritical norm of $L^{\infty}\left([0, \tau), \mathscr{H}^{1}\right)$ is infinite. If $\tau\left(h_{0}\right)<\infty$, then for for every $\alpha \in\left(\frac{1}{2}, \frac{9}{2}\right)$ the following critical norms are infinite

$$
\int_{0}^{\tau\left(h_{0}\right)}\|h(s)\|_{H^{\alpha}}^{\frac{8}{2 \alpha-1}} d s=\infty
$$


Remark 15 As it is used several times in the proofs, we state the following elementary interpolation inequality. For $\gamma>\alpha$ and $\beta \in[\alpha, \gamma]$,

$$
\|h\|_{\beta} \leq C\|h\|_{\alpha}^{\frac{\gamma-\beta}{\gamma-\alpha}}\|h\|_{\gamma}^{\frac{\beta-\alpha}{\gamma-\alpha}}
$$

Note that for short-hand notation, we abbreviate $\|\cdot\|_{s}=\|\cdot\|_{\mathscr{H}^{s}}=\left\|D^{s} \cdot\right\|_{\mathscr{L}^{2}}$ with $D^{s}=$ $(-\Delta)^{s / 2}$.

Proof Fix $\alpha \in\left(\frac{1}{2}, \frac{9}{2}\right)$, then by integration by parts and the Sobolev embedding $H^{\frac{1}{6}} \subset L^{3}$,

$$
\partial_{t}\|h\|_{1}^{2}+2\|h\|_{3}^{2}=-2\left\langle\partial_{x} h, \partial_{x}^{3}\left(\left|\partial_{x} h\right|^{2}\right)\right\rangle_{L^{2}}=2 \int_{0}^{L}\left(\partial_{x}^{2} h\right)^{3} d x \leq c\|h\|_{\frac{13}{6}}^{3} .
$$

By interpolation (19) using Young's inequality,

$$
\partial_{t}\|h\|_{1}^{2}+2\|h\|_{3}^{2} \leq\|h\|_{3}^{2}+c\|h\|_{\alpha}^{\frac{8}{2 \alpha-1}}\|h\|_{1}^{2} .
$$

Finally, by Gronwall's lemma, the proof is complete.

Remark 16 Note that we can prove the same statement for the $L^{p}\left(\left[0, \tau\left(h_{0}\right)\right], Y\right)$ norm of any other space $Y$ provided

$$
\|h\|_{\frac{13}{6}}^{3} \leq\|h\|_{3}^{2}+c\|h\|_{Y}^{p}\|h\|_{1}^{2} .
$$

Thus as example we also obtain under the assumptions of Theorem 7 that also the following critical norms are infinite:

$$
\int_{0}^{\tau\left(h_{0}\right)}\|h(s)\|_{W^{1,4}}^{16 / 3} d s=\infty \quad \text { and } \quad \int_{0}^{\tau\left(h_{0}\right)}\|h(s)\|_{C^{1}}^{4} d s=\infty .
$$

Moreover, we can consider derivatives of other $\mathscr{H}^{s}$-norms. Thus in principle we can obtain any critical space with this approach.

\subsection{Blow up below criticality}

In this section we will study the blow up in a space below criticality, i.e. in some $\mathscr{H}^{s}$ with $s<\frac{1}{2}$. This is a slight generalisation of Theorem 7 and prepares the results of Leray type shown later. For $\frac{1}{4} \leq s \leq 1$ we obtain:

$$
\begin{aligned}
\partial_{t}\|h\|_{s}^{2} & \leq-c\|h\|_{2+s}^{2}+4 \int_{0}^{L} D^{2 s} \partial_{x} h \cdot \partial_{x} h \partial_{x}^{2} h d x \\
& \leq-c\|h\|_{2+s}^{2}+C\|h\|_{1+2 s}\|h\|_{\frac{9}{4}}\|h\|_{\frac{5}{4}},
\end{aligned}
$$

where we used the Sobolev embedding $H^{\frac{1}{4}} \subset L^{4}$. Fix $\gamma \in\left(\frac{1}{2}, \frac{5}{4}\right]$ and use (19)

$$
\partial_{t}\|h\|_{s}^{2} \leq-c\|h\|_{2+s}^{2}+C\|h\|_{\gamma}^{\frac{3+2 s}{4+2 s-2 \gamma}}\|h\|_{2+s}^{\frac{9+4 s-6 \gamma}{4+2 s-2 \gamma}} .
$$

Using Young's inequality with exponents $p=(8+4 s-4 \gamma) /(9+4 s-6 \gamma)$ and $q=(8+4 s-$ $4 \gamma) /(2 \gamma-1)$ we derive

$$
\partial_{t}\|h\|_{s}^{2} \leq C\|h\|_{\gamma}^{\frac{2(3+2 s)}{2 \gamma-1}} .
$$

This inequality implies the following result. 
Theorem 8 Under Assumption 6 suppose $s \in\left[\frac{1}{4}, 1\right]$. Then for $\gamma \in\left(\frac{1}{2}, \frac{5}{4}\right]$ :

$$
\int_{0}^{\tau\left(h_{0}\right)}\|h(t)\|_{\gamma}^{\frac{2(3+2 s)}{2 \gamma-1}} d t=\infty
$$

Note that for a blow up below criticality with $s<\frac{1}{2}$ the $L^{p}\left([0, T], H^{\gamma}\right)$-norm in this theorem has a smaller $p$ than assured by Theorem 7. For fixed $s$ the spaces in the above theorem always have the same level of criticality.

\subsection{Leray type results}

We state the following theorem, which is based on one of the several celebrated results of Leray [70] on the Navier-Stokes equations. This relies mainly on a comparison result for ODEs and energy estimates.

Theorem 9 Under Assumption 6. If $s>\frac{1}{2}$, then there is a universal constant $C>0$ only depending on such that

$$
\|h(t)\|_{s} \geq C\left(\tau\left(h_{0}\right)-t\right)^{-(2 s-1) / 8} \quad \text { for all } t \in\left[0, \tau\left(h_{0}\right)\right) .
$$

Proof For $s=1+\delta$ with $\delta \in\left(-\frac{1}{2}, \frac{3}{2}\right)$

$$
\begin{aligned}
\partial_{t}\|h\|_{1+\delta}^{2}+2\|h\|_{3+\delta}^{2} & =-2 \int_{0}^{L} \partial_{x}^{2} D^{2 \delta} h \cdot B(h, h) d x=4 \int_{0}^{L} \partial_{x} D^{2 \delta} h \cdot B\left(h, \partial_{x} h\right) d x \\
& \leq C|h|_{1+\delta+\varepsilon}|h|_{\frac{5}{2}-\varepsilon}|h|_{3+\delta},
\end{aligned}
$$

where we used Lemma 1 with $\alpha=2+\delta, \beta=\frac{1}{2}-\varepsilon$, and $\gamma=-\alpha+\varepsilon$ for some small $\varepsilon \in\left(0, \frac{1}{2}\right)$ such that $\varepsilon+\delta \in\left(-\frac{1}{2}, \frac{3}{2}\right)$. Now interpolation (see Remark 15) yields

$$
\partial_{t}\|h\|_{1+\delta}^{2}+2\|h\|_{3+\delta}^{2} \leq C\|h\|_{1+\delta}^{\frac{1}{4}(7-2 \delta)} \mid h \|_{3+\delta}^{\frac{1}{4}(5+2 \delta)} .
$$

As $(5+2 \delta)<8$, apply Young's inequality with $p=8 /(7-2 \delta)$ and $q=8 /(1+2 \delta)$ to derive

$$
\partial_{t}\|h\|_{1+\delta}^{2} \leq C\|h\|_{1+\delta}^{2(5+2 \delta) /(1+2 \delta)}=C\|h\|_{s}^{2(3+2 s) /(2 s-1)} .
$$

Recall that by comparison principle for ODEs for a solution $\varphi$ of $\varphi^{\prime} \leq C \varphi^{p}$, on $(0, \tau)$ we have

$$
\varphi(s) \leq\left[\varphi(t)^{-(p-1)}+C(p-1)(t-s)\right]^{-\frac{1}{p-1}} \quad \text { for all } 0<t<s<\tau\left(h_{0}\right)
$$

Solving this for $\varphi(t)$ and passing to the limit $s \rightarrow \tau\left(h_{0}\right)$ yields the claim for $s \in\left(\frac{1}{2}, \frac{5}{2}\right)$.

The general case is proved similarly, by starting with $\partial_{t}\|h\|_{k+\delta}^{2}$ for $k \in \mathbf{N}$ and then distributing the derivatives as evenly as possible on the tri-linear terms by integration by parts. Thus proceeding as above yields the claim. 
6.5 The set of singular times

Let us state without proof a bound on the set of singular times. Note that due to the energy estimate from (3) solutions are at some point smooth and small in $\mathscr{H}^{2}$ and thus from that point on one has a global unique solution.

Let $h$ be a global weak solution to (9) and consider the set of regular times of $h$,

$$
\mathscr{R}=\left\{t \in(0, \infty): h \text { is continuous with values in } \mathscr{H}^{1} \text { in a neighbourhood of } t\right\} .
$$

By the smoothness of local solutions $\mathscr{R}$ is equal to the set of all times $t$ such that $h$ is $C^{\infty}$ in space and time in a neighbourhood of $t$. Define the set of singular times $\mathscr{S}=[0, \infty) \backslash \mathscr{R}$.

The theorem (in the spirit of results of Leray [70], Scheffer [100] for Navier-Stokes) states, that the set of singular times is "small". For the proof see [16].

Theorem 10 Given a global weak solution $h$ to (9), the set $\mathscr{S}$ of singular times of $h$ is a compact subset of $[0, \infty)$ and

$$
\mathbb{H}^{\frac{1}{4}}(\mathscr{S})=0
$$

where $\mathbb{H}^{\frac{1}{4}}$ is the $\frac{1}{4}$-dimensional Hausdorff measure.

Remark 17 It is possible to give a further limit to the set of singular times by showing that its box-counting dimension is smaller or equal than $\frac{1}{4}$. This result can be proved as in [90] and we refer to this paper for further details.

\subsection{An "inviscid" variation}

Analogous to the relation Navier-Stokes and Euler equation, one might neglect the surface diffusion $h_{x x x x}$ and keeps only the nonlinear surface dynamics. But in this case the blow-up is easier to prove. Consider

$$
\partial_{t} h+\left(h_{x}^{2}\right)_{x x}=0
$$

subject to periodic boundary conditions on $[0, L]$. If we have a non-constant smooth solution of the above problem, then we will show below that there is always a blow-up in finite time.

In fact the main issue for the inviscid problem above is the existence of a, at least local, smooth solution. By similarity with the Navier-Stokes/Euler equations, one may believe that the methods developed in the latter case $[111,64]$ may work. A quick series of computations shows that this is not the case. Indeed there is a more fundamental problem here, since not even general results such as the Cauchy-Kovalevskaya theorem work. The reason, roughly speaking, is the number of derivatives in the nonlinearity.

It is easy to verify that for a sufficiently smooth solution $h$ the following quantities

$$
\mathscr{M}_{0}:=\int_{0}^{L} h(t, x) d x, \quad \mathscr{E}_{0}:=\int_{0}^{L} h(t, x)^{2} d x, \quad \text { and } \quad \int_{0}^{L} h_{x}(t, x)^{3} d x,
$$

are conserved and independent of time. As usual we can assume without loss of generality that the total mass $\mathscr{M}_{0}$ is zero.

Let us prove first a bound in $H^{1}$. Let $\phi(x)$ be a smooth function such that $\phi^{\prime \prime}(x)=$ $\arctan (x)$, then the energy estimate for $\phi(h)$ yields

$$
\frac{2}{3} \int_{0}^{t} \int_{0}^{L} \frac{h_{x}^{4}(\tau)}{1+h^{2}(\tau)} d x d \tau=\int_{0}^{L} \phi(h(0)) d x-\int_{0}^{L} \phi(h(t)) d x .
$$


Since $\phi$ has quadratic growth, the integrals on the right-hand side are uniformly bounded in time by a constant depending on $\mathscr{E}_{0}$. In particular,

$$
\begin{aligned}
\int_{0}^{L} h_{x}(t)^{2} d x & \leq\left(\int_{0}^{L} \frac{h_{x}(t)^{4}}{1+h(t)^{2}} d x\right)^{\frac{1}{2}}\left(\int_{0}^{L} 1+h(t)^{2} d x\right)^{\frac{1}{2}} \\
& \leq c\left(L, \mathscr{E}_{0}\right)\left(\int_{0}^{L} \frac{h_{x}(t)^{4}}{1+h(t)^{2}} d x\right)^{\frac{1}{2}}
\end{aligned}
$$

and we can conclude that for every $t>0$,

$$
\int_{0}^{t}\left\|h_{x}(\tau)\right\|_{L^{2}}^{2} d \tau \leq C\left(L, \mathscr{E}_{0}\right) .
$$

The $H^{1}$ estimate immediately yields a $L^{\infty}$ bound. Due to Sobolev's embeddings,

$$
\int_{0}^{\infty}\|h(t)\|_{L^{\infty}}^{2} d t \leq C\left(L, \mathscr{E}_{0}\right) .
$$

We can now preliminarily show that some solutions cannot be global.

Proposition 6 Assume that $h(0)$ is smooth and non-zero. If

$$
\int_{0}^{L} h(0)^{3} d x \leq 0
$$

then there cannot be a global smooth solution with initial condition $h(0)$.

Proof Let us first remark that $h$ is by assumption on $h(0)$ also non-zero. The energy estimate for $\int h^{3} d x$ yields after a short calculation

$$
4 \int_{0}^{t} \int_{0}^{L}\left|h_{x}(s)\right|^{4} d x d s=\int_{0}^{L} h(0)^{3} d x-\int_{0}^{L} h(t)^{3} d x \leq \int_{0}^{L} h(0)^{3} d x+\mathscr{E}_{0}\|h(t)\|_{\infty} .
$$

By (20) we can deduce that there is a sequence $t_{n} \uparrow \infty$ such that $\left\|h\left(t_{n}\right)\right\|_{L^{\infty}} \rightarrow 0$. If we apply this fact to the previous inequality, we obtain a contradiction to $h$ being non-zero:

$$
4 \int_{0}^{\infty} \int_{0}^{L}\left|h_{x}(s)\right|^{4} d x d s \leq \int_{0}^{L} h(0)^{3} d x \leq 0
$$

Let us prove now that no non-zero global solution exist.

Theorem 11 Every smooth solution that does not blow up in finite time is constant.

Proof Assume, as before, without loss of generality that $\mathscr{M}_{0}=0$. A simple computation yields

$$
\frac{d}{d t} \int_{0}^{L} \mathrm{e}^{-h(t)} d x=\frac{1}{3} \int_{0}^{L} h_{x}^{4} \mathrm{e}^{-h(t)} d x,
$$

and hence for any $t \geq s \geq 0$

$$
\int_{0}^{L} \mathrm{e}^{-h(s)} d x \leq \int_{0}^{L} \mathrm{e}^{-h(t)} d x .
$$


Consider again the sequence of times $t_{n} \uparrow \infty$ such that $\left\|h\left(t_{n}\right)\right\|_{L^{\infty}} \rightarrow 0$. On this sequence

$$
\int_{0}^{L} \mathrm{e}^{-h\left(t_{n}\right)} d x \rightarrow L \quad \text { for } \rightarrow \infty .
$$

Therefore by the conservation of mass for any $s \geq 0$

$$
\frac{1}{L} \int_{0}^{L} \mathrm{e}^{-h(s)} d x \leq \lim _{n \rightarrow \infty} \frac{1}{L} \int_{0}^{L} \mathrm{e}^{-h\left(t_{n}\right)} d x=1=\mathrm{e}^{-\frac{1}{L} \int_{0}^{L} h(s) d x} .
$$

This is the case of equality in the Jensen inequality, thus $h(s)$ is constant and, since $\mathscr{M}_{0}=0$, $h(s) \equiv 0$.

\section{Complex-valued blow up}

In this section we study complex-valued solutions of the equation posed in $d=1$. Obviously, our energy estimate (3) that is used for global existence fails. Our main aim is to provide a simple proof, that there is a blow up for complex valued solutions of (9). Here we construct a large class of solutions that do blow up. The key idea is to derive an infinite-dimensional system of ODEs that allows blow up by transporting mass to $\infty$ in finite time. This is also the idea of the dyadic model embedded into an averaged Navier-Stokes equation in [113].

Our result verifies that most energy estimates, which are usually also true for complexvalued solutions, cannot be used alone to rule out a possible blow up.

\subsection{The network of ODEs}

We rewrite the PDE as an infinite system of ODEs, by going into Fourier-space. Suppose for simplicity of presentation that the equation is posed on $[0,2 \pi]$. Let

$$
h(t, x)=\sum_{k=-\infty}^{\infty} h_{k}(t) \mathrm{e}^{i x k}
$$

then for all $k \neq 0$ (note, $h_{0} \equiv 0$ by mass conservation)

$$
\partial_{t} h_{k}=-k^{4} h_{k}+k^{2} \sum_{l=-\infty}^{\infty} l h_{l}(k-l) h_{k-l}
$$

Definition 7 Define the weighted Fourier modes by $\chi_{k}=k h_{k}$.

Thus we have for all $k \neq 0$

$$
\partial_{t} \chi_{k}=-k^{4} \chi_{k}+k^{3} \sum_{l=-\infty}^{\infty} \chi_{l} \chi_{k-l} .
$$

In the following, we need local existence and uniqueness of solutions only for a simplified system, where it will be obvious. But let us remark that either the results in [19] for a probabilistic representation via branching processes yields the existence of solutions for the system (21), or we can modify our results in Section 3 and use existence of solutions for the original PDE. 
Definition 8 We define the following spaces

$$
\begin{gathered}
\Sigma=\left\{\chi \in \mathbf{C}^{\mathbf{Z}}: \chi_{k}=0 \forall k \leq 0\right\} \cong \mathbf{C}^{\mathbf{N}}, \\
\Sigma^{+}=\left\{\chi \in \Sigma: \chi_{k} \geq 0 \forall k \geq 0\right\} \cong[0, \infty)^{\mathbf{N}}, \\
\Sigma(L)=\left\{\chi \in \Sigma:\left|\chi_{k}\right| \leq L^{k} \forall k \geq 0\right\} \text { for } L>0 .
\end{gathered}
$$

The space $\Sigma(L)$ is a weighted $L^{\infty}$-space in Fourier space for solutions $h$ of (9). Note that $\left|\chi_{k}\right| \leq L^{k}$ implies $\left|h_{k}\right| \leq L^{k} / k$. For $L>1$ this is a space of quite irregular distributions, while for $L \in(0,1)$ the corresponding functions $h$ are $C^{\infty}$.

For $L=1$ we conjecture that this corresponds to a critical space for $h$.

Lemma 9 The spaces $\Sigma$ and $\Sigma^{+}$are invariant for (21) for sufficiently smooth solutions.

Proof (Idea of Proof) Probabilistic representation of [19] gives invariance of a unique solution among all sufficiently regular solutions. The existence of invariant solutions also follows from the discussion in the next subsection.

\subsection{Explicit solution}

In $\Sigma$ Equation (21) simplifies to

$$
\partial_{t} \chi_{k}=-k^{4} \chi_{k}+k^{3} \sum_{l=1}^{k-1} \chi_{l} \chi_{k-l} \quad \text { for all } k>0 .
$$

Thus any Fourier mode $k$ is driven only by lower modes $l \in\{1, \ldots, k\}$. For the first few equations:

$$
\left\{\begin{array}{l}
\partial_{t} \chi_{1}=-\chi_{1} \\
\partial_{t} \chi_{2}=-16 \chi_{2}+8 \chi_{1}^{2} \\
\partial_{t} \chi_{3}=-81 \chi_{3}+54 \chi_{1} \chi_{2}, \\
\partial_{t} \chi_{4}=-216 \chi_{4}+108 \chi_{1} \chi_{3}+54 \chi_{2}^{2} \\
\ldots
\end{array}\right.
$$

This system can be solved inductively, and we obtain easily:

Lemma 10 For all initial conditions in $\Sigma$, there is a solution $\chi$ of (22) such that $\chi(t) \in \Sigma$ for all $t \geq 0$. Moreover, for all such solutions, the space $\Sigma^{+}$of positive solutions is invariant.

Let us remark, that there is no regularity for the solution provided by this Lemma.

Remark 18 (Mild solution) We can also restate (22) as mild formulation for solutions in $\Sigma$.

$$
\chi_{k}(t)=\mathrm{e}^{-t k^{4}} \chi_{k}(0)+k^{3} \sum_{l=1}^{k-1} \int_{0}^{t} \mathrm{e}^{-(t-s) k^{4}} \chi_{l}(s) \chi_{k-l}(s) d s .
$$


7.3 Exponential Bound

This section provides the invariance of $\Sigma(L)$.

Lemma 11 Suppose that $\chi$ solves (22) in $\Sigma$ and $\left|\chi_{k}(0)\right| \in \Sigma(L)$ for some $L>0$, then $\chi_{k}(t) \in$ $\Sigma(L)$ for all $t>0, k>0$.

This allows for exponential divergence (in $k$ ) of solutions provided $\chi_{k}(0)>1$ for one $k$.

Proof By induction. First, $\left|\chi_{1}(t)\right|=\mathrm{e}^{-t}\left|\chi_{1}(0)\right| \leq L$. Then, from the mild formulation (using the assertion up to $k-1$ )

$$
\begin{aligned}
\left|\chi_{k}(t)\right| & \leq \mathrm{e}^{-t k^{4}}\left|\chi_{k}(0)\right|+k^{3} \int_{0}^{t} \mathrm{e}^{-(t-s) k^{4}} \sum_{l=1}^{k-1}\left|\chi_{l}(s)\right| \cdot\left|\chi_{k-l}(s)\right| d s \\
& \leq \mathrm{e}^{-t k^{4}} L^{k}+k^{3} \int_{0}^{t} \mathrm{e}^{-(t-s) k^{4}} \sum_{l=1}^{k-1} L^{l} L^{k-l} d s \\
& \leq L^{k}\left(\mathrm{e}^{-t k^{4}}+k^{4} \int_{0}^{t} \mathrm{e}^{-(t-s) k^{4}} d s\right) \leq L^{k} .
\end{aligned}
$$

Note that the Lemma is also true if we consider only the first finitely many $k$.

\subsection{Stability}

Under very weak regularity assumptions (even exponentially growth of Fourier-coefficients) we can prove that the solution is eventually smooth after some time and converges to 0 .

Lemma 12 Suppose that $\chi$ solves (22) in $\Sigma(L)$ for some $L>0$. that

Then for all small $\varepsilon>0$ there is a sequence of times $0<T_{1}<\ldots<T_{k} \nearrow T^{*}<\infty$, such

$$
\left|\chi_{k}(t)\right| \leq \frac{1}{2} \varepsilon^{k} \quad \text { for all } t>T_{k} \text { and } k>0
$$

Proof By Induction. First,

$$
\left|\chi_{1}(t)\right| \leq \mathrm{e}^{-t} L<\frac{1}{2} \varepsilon \quad \text { for } t>t_{1}=\ln (2 L / \varepsilon) .
$$

Then, using the mild formulation (23)

$$
\begin{aligned}
\chi_{k}\left(T_{k-1}+t\right) & \leq \mathrm{e}^{-t k^{4}}\left|\chi_{k}\left(T_{k-1}\right)\right|+k^{3} \int_{0}^{t} \mathrm{e}^{-(t-s) k^{4}} \sum_{l=1}^{k-1} \frac{1}{2} \varepsilon^{l} \frac{1}{2} \varepsilon^{k-l} d s \\
& \leq \mathrm{e}^{-t k^{4}} L^{k}+\frac{1}{4} \varepsilon^{k}<\frac{1}{2} \varepsilon^{k}
\end{aligned}
$$

for all $t>t_{k}=\frac{1}{k^{4}} \ln \left(4 L^{k} / \varepsilon^{k}\right)$. Finally, define $T_{k}=\sum_{l=1}^{k} t_{l}$, which converges as $t_{l} \sim C / l^{3}$. 


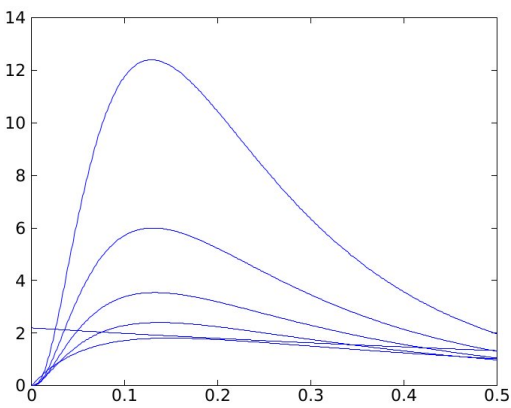

Fig. 2 First six curves of $\chi_{k}$, growing fast in $k$ already for $\chi_{1}(0)=2.2$ and $\chi_{k}(0)=0$ for $k \neq 1$. The value necessary for the lower bound of Theorem 12 would be $\chi_{1}(0)>27$, as $a=b=1$.

\subsection{The Blow-up}

This section provides a very simple lower bound that leads to a blow up for sufficiently large smooth solutions in $\Sigma^{+}$. Let us remark that $[71,72]$ proved a similar result for 3D-Navier Stokes which is significantly more complicated, but yields solution with finite $\mathscr{L}^{2}$-norm, while we only obtain a lower bound on the blow up of solutions.

Theorem 12 Suppose $\chi$ is a solution of (22) in $\Sigma^{+}$, such that for some $a, b \in \mathbf{N}$ and $C>0$

$$
\chi_{k}(0) \geq C \quad \text { for } k \in[a, b] .
$$

Then for any $L \in\left[2, \frac{2}{27} \frac{\mathrm{Ca}^{3}}{\mathrm{~b}^{4}}\right]$ (and thus $C>0$ large) there are times $0=T_{0}<T_{1}<\ldots<T_{k} \nearrow$ $T^{*}<\infty$ such that for all $N \in \mathbf{N}$, the following is true:

For all $k \in\left[a 2^{N}, b 2^{N}\right]$

$$
\chi_{k}\left(T_{N}\right) \geq C L^{N} \quad \text { and } \quad \inf _{t \in\left[T_{N}, T_{N+1}\right]}\left\{\chi_{k}(t)\right\} \geq \frac{2}{3} C L^{N} .
$$

Thus for some time $t^{*} \leq T^{*}$

$$
\|\chi(t)\|_{\infty}=\sup _{k \in \mathbf{N}}\left|\chi_{k}(t)\right| \nearrow_{\infty} \text { for } t \rightarrow t^{*} .
$$

Note that this result is in well agreement with criticality. It is easy to check that $\|\chi(t)\|_{\infty} \leq$ $C\|h\|_{\mathscr{H}^{s}}$ for all $s \geq \frac{1}{2}$. Thus the norm blows up in the critical and all subcritical $\mathscr{H}^{s}$-spaces.

Proof We proceed by induction on $N$. Define $T_{N}=\sum_{k=1}^{N} t_{k}$, where $t_{k}$ is defined later. Furthermore, fix $\alpha=\frac{2}{3}$.

For $N=0$ the lower bound holds at $T_{0}=0$. For the lower bound on $\left[T_{0}, T_{1}\right]=\left[0, t_{1}\right]$ consider for $k \in[a, b]$ that for $t \in\left[0, t_{1}\right]$

$$
\chi_{k}(t) \geq \mathrm{e}^{-t k^{4}} \chi_{k}(0) \geq \mathrm{e}^{-t_{1} b^{4}} C=\alpha C \quad \text { if } t_{1}=\frac{1}{b^{4}} \ln \left(\alpha^{-1}\right) .
$$

For the induction step from $N-1$ to $N$ we first prove the bound at $T_{N}$. Fix $k \in[a, b] \cdot 2^{N}$. By assumption on $\left[T_{N-1}, T_{N}\right]$ we have

$$
\chi_{l} \chi_{k-l} \geq \alpha^{2} C^{2} L^{2 N-2}
$$


for at least one $l \in\{1, \ldots, k-1\}$. Thus

$$
\begin{aligned}
\chi_{k}\left(T_{N}\right) & =\chi_{k}\left(T_{N-1}+t_{N}\right) \\
& \geq k^{3} \int_{0}^{t_{N}} \mathrm{e}^{-\left(t_{N}-s\right) k^{4}} \sum_{l=1}^{k-1} \chi_{l}\left(T_{N-1}+s\right) \chi_{k-l}\left(T_{N-1}+s\right) d s \\
& \geq a^{3} 2^{3 N} \int_{0}^{t_{N}} \mathrm{e}^{-\left(t_{N}-s\right) b^{4} 2^{4 N}} d s \cdot \alpha^{2} C^{2} L^{2 N-2} \\
& \geq \frac{a^{3}}{b^{4}} \frac{1}{2^{N}}\left(1-\mathrm{e}^{-t_{N} b^{4} 2^{4 N}}\right) \alpha^{2} C^{2} L^{2 N-2}=C L^{N}
\end{aligned}
$$

provided that

$$
t_{N}=\frac{1}{b^{4} 2^{4 N}} \ln \left(\left(1-\left(\frac{2}{L}\right)^{N} \frac{b^{4} L^{2}}{C \alpha^{2} a^{3}}\right)^{-1}\right)
$$

Here we used that $t_{N}$ is well defined for all $N$, if $L \geq 2$ and $2 L b^{4}<C \alpha^{2} a^{3}$, which is true by assumption.

On $\left[T_{N}, T_{N+1}\right]$ we now use the lower bound at time $T_{N}$. Fix again $k \in[a, b] 2^{N}$, then

$$
\chi_{k}\left(T_{N}+t\right) \geq \mathrm{e}^{-t k^{4}} \chi_{k}\left(T_{N}\right) \geq \mathrm{e}^{-t b^{4} 2^{4 N}} C L^{N} \geq \alpha C L^{N},
$$

if $t_{N} \leq b^{-4} 2^{-4 N} \ln \left(\alpha^{-1}\right)$. This in turn is true for all $N$, if

$$
\frac{1}{2}(1-\alpha) \alpha^{2} \geq \frac{L b^{4}}{C a^{3}}
$$

which is true by assumption. Note that $\alpha=\frac{2}{3}$ maximizes the right hand side with maximum $2 / 27$. It remains to check that $T_{*}=\sum_{l=1}^{\infty} t_{l}<\infty$. But this is true, as for $N \rightarrow \infty$

$$
t_{N}=\frac{1}{b^{4} 2^{4 N}} \ln \left(1+\mathscr{O}\left(\left(\frac{2}{L}\right)^{N}\right)\right)=\frac{1}{b^{4} 2^{4 N}} \mathscr{O}\left(\left(\frac{2}{L}\right)^{N}\right)=\mathscr{O}\left(\left(\frac{1}{8 L}\right)^{N}\right) .
$$

Acknowledgements We thank Christian Nolde for the code used in the simulations presented in Figure 1. We also like to thank Franco Flandoli for joint work on the inviscid surface growth, Martin Hairer for many interesting discussions, and Michael Winkler for discussions on possible blow up.

\section{References}

1. R. A. Adams, J. J. F. Fournier Sobolev Spaces, Academic Press, 2003.

2. L. AGÉLAS, Global regularity of solutions of equation modeling epitaxy thin film growth in $\mathbb{R}^{d}, d=1,2$ J. Evolution Eq., online first (2014)

3. A. L. Barabasi, H. E. Stanley, Fractal concepts in surface growth, Cambridge University Press, 1995.

4. D. Barbato, F. Morandin, M. Romito, Smooth solutions for the dyadic model, Nonlinearity 24 (2011), no. 11, 3083-3097, (featured article).

5. D. BARbato, F. Morandin, M. Romito, Global regularity for a logarithmically supercritical hyperdissipative dyadic equation, Dyn. Partial Differ. Equ. 11 (2014), no. 1, 39-52.

6. D. BARBATO, F. MORANDIN, M. ROMITO, Global regularity for a slightly supercritical hyperdissipative Navier-Stokes system, Analysis and PDE 7 (2014), no. 8, 2009-2027.

7. J. T. Beale, T. Kato, A. MajdA, Remarks on the breakdown of smooth solutions for the 3-D Euler equations, Comm. Math. Phys. 94, no. 1 (1984), 61-66.

8. D. BLÖMKER, Nonhomogeneous noise and $Q$-Wiener processes on bounded domains, Stoch. Anal. Appl. 23 (2005), no. 2, 255-273. 
9. D. BlÖMKER, F. Flandoli, M. Romito, Markovianity and ergodicity for a surface growth PDE, Ann. Probab. 37 (2009), no. 1, 275-313.

10. D. BlÖMKER, C. GugG, Thin-film-growth-models: On local solutions, Albeverio, Sergio (ed.) et al., Recent developments in stochastic analysis and related topics. Proceedings of the first Sino-German conference on stochastic analysis (a satellite conference of ICM 2002), Beijing, China, 29 August - 3 September 2002. River Edge, NJ: World Scientific. 66-77 (2004)., 2004.

11. D. BlÖMKER, C. GUGG, On the existence of solutions for amorphous molecular beam epitaxy, Nonlinear Anal. Real World Appl. 3 (2002), no. 1, 61-73.

12. D. BlÖMKER, C. GugG, M. RAIBLE, Thin-film-growth models: roughness and correlation functions, European J. Appl. Math. 13 (2002), no. 4, 385-402.

13. D. Blömker, M. Hairer, Stationary solutions for a model of amorphous thin-film growth, Stoch. Anal. Appl. 22 (2004), no. 4, 903-922.

14. D. BLÖMKER, S. MAIER-PAAPE, T. WANNER Roughness in surface growth equations, Interfaces and Free Boundaries, 3, (2001), no. 4, 465-484.

15. D. Blömker, C. Nolde, J. C. Robinson, Rigorous Numerical Verification of Uniqueness and Smoothness in a Surface Growth Model. Journal of Mathematical Analysis and Applications, 429, (2015) no. 1, 311-325.

16. D. BlÖmKer, M. Romito, Regularity and blow up in a surface growth model, Dyn. Partial Differ. Equ. 6 (2009), no. 3, 227-252.

17. D. BLÖMKER, M. ROMito, Local existence and uniqueness in the largest critical space for a surface growth model, NoDEA Nonlinear Differential Equations Appl. 19 (2012), no. 3, 365-381.

18. D. Blömker, M. Romito, Local Existence and Uniqueness for a Two-Dimensional Surface Growth Equation with Space-Time White Noise, Stoch. Anal. Appl., 31(6):1049-1076, (2013).

19. D. BlÖMKer, M. Romito, R. TRIBE, A probabilistic representation for the solutions to some nonlinear PDEs using pruned branching trees, Ann. Inst. H. Poincaré Probab. Statist. 43 (2007), no. 2, 175192.

20. V. I. Bogachev, Gaussian measures, Mathematical Surveys and Monographs, vol. 62, American Mathematical Society, Providence, RI, 1998.

21. L. Caffarelli, R. Kohn, L. Nirenberg, Partial regularity of suitable weak solutions of the NavierStokes equations, Comm. Pure Appl. Math. 35 (1982), no. 6, 771-831.

22. M. CANNONE, Harmonic analysis tools for solving the incompressible Navier-Stokes equations, in Handbook of Mathematical Fluid Dynamics, vol. 3, S. Friedlander and D. Serre eds., 161-244, NorthHolland, Amsterdam, 2004.

23. M. CASTRO, R. CUERno, L. VÁZQUeZ, R. GAGO, Self-organized ordering of nanostructures produced by ion-beam sputtering, Phys. Rev. Lett. 94 (2005), 016102.

24. S. I. Chernyshenko, P. Constantin, J. C. Robinson, E. S. Titi, A posteriori regularity of the three-dimensional Navier-Stokes equations from numerical computations. J. Math. Phys., 48(6):065204, $15 \mathrm{p.},(2007)$.

25. P.-L. CHOw, Stochastic partial differential equations, Chapman \& Hall/CRC Applied Mathematics and Nonlinear Science Series. Boca Raton, FL: Chapman \& Hall/CRC. ix, 281 p., 2007.

26. P. Collet, J.-P. Eckmann, H. Epstein, J. Stubbe, A global attracting set for the KuramotoSivashinsky equation, Commun. Math. Phys. 152 (1993), no. 1, 203-214.

27. R. Cuerno, A.-L. BARABÁsi, Dynamic scaling of ion-sputtered surfaces, Phys. Rev. Lett. 74 (1995), 4746-474.

28. G. D. Prato, A. Debussche, Stochastic Cahn-Hilliard equation, Nonlinear Anal., Theory Methods Appl., 26(2):1-263, (1996).

29. G. D. Prato, A. Debussche, Two-dimensional Navier-Stokes equations driven by a space-time white noise, J. Funct. Anal. 196 (2002), no. 1, 180-210.

30. G. D. Prato, A. Debussche, Ergodicity for the 3D stochastic Navier-Stokes equations, J. Math. Pures Appl. (9) 82 (2003), no. 8, 877-947.

31. G. Da Prato, J. ZabCZYK, Stochastic equations in infinite dimensions, Encyclopedia of Mathematics and Its Applications. 44, Cambridge University Press, Cambridge, 1992.

32. G. Da PRATO, J. ZABCZYK, Stochastic equations in infinite dimensions, 2nd Edition, vol. 152 of Encyclopedia of of Mathematics and its Applications. Cambridge University Press, Cambridge, 2014.

33. G. DA PRATO, J. ZABCZYK, Ergodicity for infinite-dimensional systems, London Mathematical Society Lecture Note Series, 229. Cambridge University Press, Cambridge, 1996.

34. M. DAshti, J. C. Robinson, An a posteriori condition on the numerical approximations of the NavierStokes equations for the existence of a strong solution. SIAM J. Numer. Anal., 46(6):3136-3150, (2008).

35. A. Debussche, C. OdAsso, Markov solutions for the 3D stochastic Navier-Stokes equations with state dependent noise, J. Evol. Equ. 6 (2006), no. 2, 305-324. 
36. C. Escudero, F. Gazzola, I. Peral, Global existence versus blow-up results for a fourth order parabolic PDE involving the Hessian. Journal de Mathématiques Pures et Appliquées. In Press, 2014.

37. C. Escudero, R. HAKL, I. Peral, P. J. Torres, On radial stationary solutions to a model of nonequilibrium growth. Euro. J. of Appl. Math. 24 (2013), no.3, 437-453.

38. A. ES-SARHIR, W. STANNAT, Improved moment estimates for invariant measures of semilinear diffusions in Hilbert spaces and applications. Journal of Functional Analysis, 259, (2010), no.5, 1248-1272.

39. F. Flandoli, Irreducibility of the 3-D stochastic Navier-Stokes equation, J. Funct. Anal. 149 (1997), no. $1,160-177$.

40. F. Flandoli, An introduction to 3D stochastic fluid dynamics, Proceedings of the CIME course on SPDE in hydrodynamics: recent progress and prospects, Lecture Notes in Math., 1942, 2008, pp 51-150, Springer.

41. F. Flandoli, M. Romito, Statistically stationary solutions to the 3-D Navier-Stokes equations do not show singularities, Electron. J. Probab. 6 (2001), no. 5, 15 pp. (electronic).

42. F. Flandoli, M. Romito, Markov selections and their regularity for the three-dimensional stochastic Navier-Stokes equations, C. R. Math. Acad. Sci. Paris, Ser. I 343 (2006), 47-50.

43. F. Flandoli, M. Romito, Regularity of transition semigroups associated to a $3 D$ stochastic NavierStokes equation, Stochastic differential equations: theory and applications (Peter H. Baxendale and Sergey V. Lototski, eds.), Interdiscip. Math. Sci., vol. 2, World Sci. Publ., Hackensack, NJ, 2007, pp. 263280.

44. F. Flandoli, M. Romito, Markov selections for the three-dimensional stochastic Navier-Stokes equations, Probab. Theory Related Fields 140 (2008), no. 3-4, 407-458.

45. H. Fujita, T. Kato, On the Navier-Stokes initial value problem. I, Arch. Rational Mech. Anal. 16 (1964), 269-315.

46. T. FRISCH, A. VERGA, Effect of step stiffness and diffusion anisotropy on the meandering of a growing vicinal surface, Phys. Rev. Lett., 96:166104, (2006).

47. P. Friz, M. HAIRER, A Course on Rough Paths: With an Introduction to Regularity Structures, Springer, (2014).

48. P. Germain, N. Pavlović, G. Staffilani, Regularity of solutions to the Navier-Stokes equations evolving from small data in $B M O^{-1}$, Int. Math. Res. Not. IMRN, (21):Art. ID rnm087, 35 pp., (2007).

49. B. Goldys, M. Röckner, X. ZHANG, Martingale solutions and Markov selections for stochastic partial differential equations. Stochastic Processes Appl., 119, (2009), no. 5, 1725-1764.

50. M. Gubinelli, P. Imkeller, N. Perkows Ki, Paraproducts, rough paths and controlled distributions, 2014, arXiv:1210.2684v3 [math.PR].

51. M. HAIRER, An Introduction to Stochastic PDEs. Lecture Notes, 2009, arXiv:0907.4178 [math.PR].

52. M. HAIRER, Solving the KPZ equation, Ann. Math. 178, (2013), no. 2, 559-664.

53. M. HAIRER, A theory of regularity structures, Inventiones mathematicae, 198, (2014), no. 2, $269-504$.

54. M. HAIRER H. WEBER, Rough Burgers-like equations with multiplicative noise, Probab. Theory Related Fields 155 (2013), no. 1-2, 71-126.

55. M. HAIRER H. WEBER, Large deviations for white-noise driven, nonlinear stochastic PDEs in two and three dimensions. Preprint, (2014), arXiv:1404.5863v2 [math.PR]

56. T. Halpin-Healy, Y. C. Zhang, Kinetic roughening phenomena, stochastic growth, directed polymers and all that, Physics Reports, 254 (1995), 215-414.

57. D. HENRY, Geometric theory of semilinear parabolic equations, Lecture Notes in Mathematics, vol. 840, Springer-Verlag, Berlin, 1981.

58. R. Hoppe, S. LinZ, W. Litvinov, On solutions of certain classes of evolution equations for surface morphologies, Nonlinear Phenomena in Complex Systems, 6, (2003), 582-591.

59. R. Hoppe, E. NASH, A combined spectral element/finite element approach to the numerical solution of a nonlinear evolution equation describing amorphous surface growth of thin films, J. Numer. Math., 100, (2002), no.2, 127-136.

60. R. H. HOPPE, E. NASH, Numerical solution of a nonlinear evolution equation describing amorphous surface growth of thin films, Feistauer, M. (ed.) et al., Numerical mathematics and advanced applications. Proceedings of ENUMATH 2003, the 5th European conference on numerical mathematics and advanced applications, Prague, Czech Republic, August 18-22, 2003. Berlin: Springer. 440-448 (2004)., 2004.

61. N. IKedA, S. WATAnaBe, Stochastic differential equations and diffusion processes, North Holland Mathematical Library 24, North Holland/Kodansha 1989.

62. M.Kardar, G. PArisi, Y.-C. Zhang, Dynamic scaling of growing interfaces, Phys. Rev. Lett. 56, (1986), no. 9, 889-892.

63. I. Karatzas, S. E. Shreve, Brownian motion and stochastic calculus. 2nd ed. Graduate Texts in Mathematics, 113. Springer-Verlag. 470 pp., 1991.

64. T. KATO, Nonstationary flows of viscous and ideal fluids in $\mathbf{R}^{3}$, J. Funct. Anal. 9 (1972), 296-305. 
65. H. Koch, D. TATARU, Well-posedness for the Navier-Stokes equations, Adv. Math., 157 (2001), no. 1, $22-35$.

66. H. KосH, T. LAmM, Geometric flows with rough initial data, Asian J. Math. 16, (2012), no. 2, $209-235$.

67. N. V. KRYLOV, The selection of a Markov process from a Markov system of processes, and the construction of quasi-diffusion processes (Russian), Izv. Akad. Nauk SSSR Ser. Mat. 37 (1973), 691-708.

68. Z.-W. LAI, S. DAS SARMA, Kinetic growth with surface relaxation: Continuum versus atomistic models, Phys. Rev. Lett. 66 (1991), 2348-2351.

69. P. G. LemariÉ-RIEUSSET, Recent developments in the Navier-Stokes problem, Chapman \& Hall/CRC research notes in mathematics series 431, CRC Press, Boca Raton, Florida, 2002.

70. J. LeRAY, Sur le mouvement d'un liquide visqueux emplissant l'espace, Acta Math. 63 (1934), no. 1, 193-248.

71. D. LI, YA. G. SINAI, Blow ups of complex solutions of the 3D Navier-Stokes system and renormalization group method, J. Eur. Math. Soc. 10 (2008), no. 2, 267-313.

72. D. LI, YA. G. SinAI, Complex singularities of solutions of some $1 D$ hydrodynamic models, Physica D 237 (2008), 1945-1950.

73. K. LIU, Stability of infinite dimensional stochastic differential equations with applications, Chapman \& Hall/CRC Monographs and Surveys in Pure and Applied Math. 135. CRC Press, Boca Raton, Florida, 2006.

74. W. LIU, M. RÖCKNER, Local and global well-posedness of SPDE with generalized coercivity conditions. Journal of Differential Equations. 254 (2013), no. 2, 725-755.

75. A. LUNARDI, Analytic semigroups and optimal regularity in parabolic problems, Birkhäuser Verlag, Basel, 1995

76. C. Morosi, L. Pizzocchero, On approximate solutions of semilinear evolution equations. II: Generalizations, and applications to Navier-Stokes equations. Rev. Math. Phys., 20(6):625-706, (2008).

77. C. Morosi, L. Pizzocchero, An $H^{1}$ setting for the Navier-Stokes equations: quantitative estimates. Nonlinear Anal., Theory Methods Appl., Ser. A, Theory Methods, 74(6):2398-2414, (2011).

78. C. Morosi, L. Pizzocchero, On approximate solutions of the incompressible Euler and NavierStokes equations. Nonlinear Anal., Theory Methods Appl., Ser. A, Theory Methods, 75(4):2209-2235, (2012).

79. P. Mörters, Y. PEREs, Brownian motion. With an appendix by Oded Schramm and Wendelin Werner. Cambridge Series in Statistical and Probabilistic Mathematics 30. Cambridge University Press 403 pp. (2010).

80. J. Muñoz-García, R. Cuerno, M. Castro, Coupling of morphology to surface transport in ionbeam-irradiated surfaces: normal incidence and rotating targets, Journal of Physics: Condensed Matter, 21(22):224020, (2009)

81. J. Muñoz-García, R. Gago, L. Vázquez, J. A. Sánchez-García, R. Cuerno, Observation and modeling of interrupted pattern coarsening: Surface nanostructuring by ion erosion, Phys. Rev. Lett., 104:026101, (2010).

82. E. Nelson, The free Markoff field, J. Functional Analysis 12 (1973), 211-227.

83. A. PAZY, Semigroups of linear operators and applications to partial differential equations, Springer, New York, 1983.

84. P. Politi, D. Ben-Avraham, From the conserved Kuramoto-Sivashinsky equation to a coalescing particles model, Physica D - Nonlinear phenomena, 238 (2009), 156-161

85. C. Prévot, M. Röckner, A Concise Course on Stochastic Partial Differential Equations. Lecture Notes in Mathematics, Springer Berlin, 2007.

86. G. Prodi, Un teorema di unicit per le equazioni di Navier-Stokes (Italian), Ann. Mat. Pura Appl. 48 (1959), no. 4, 173-182.

87. M. Raible, S. J. LinZ, P. HÄNGgi, Amorphous thin film growth: Minimal deposition equation, Phys. Rev. E, 62 (2000), 1691-1705.

88. M. RAIBLE, S. LinZ, P. HÄNGGI, Amorphous thin film growth: modeling and pattern formation, Advances in Solid State Physics, 41 (2001), 391-403.

89. M. Raible, S. G. Mayr, S. J. Linz, M. Moske, P. Hänggi, K. Samwer, Amorphous thin film growth: Theory compared with experiment, Europhys. Lett., 50 (2000), 61-67.

90. J. C. RobINSON, W. SADOWSKI, Decay of weak solutions and the singular set of the three-dimensional Navier-Stokes equations, Nonlinearity, 20 (2007), no. 5, 1185-1191.

91. M. RÖCKNER, R. ZHU, X. ZHU, Local existence and non-explosion of solutions for stochastic fractional partial differential equations driven by multiplicative noise. Stochastic Processes Appl., 124 (2014), no. 5, 1974-2002.

92. M. Romito, Analysis of equilibrium states of Markov solutions to the 3D Navier-Stokes equations driven by additive noise, J. Stat. Phys. 131 (2008), no. 3, 415-444. 
93. M. Romito, Existence of martingale and stationary suitable weak solutions for a stochastic NavierStokes system, Stochastics, 82 (2010), no. 3, 327-337.

94. M. Romito, An almost sure energy inequality for Markov solutions to the 3D Navier-Stokes equations, Stochastic Partial Differential Equations and Applications, Quad. Mat., 25, Dept. Math., Seconda Univ. Napoli, Caserta, 2010, 243-255.

95. M. Romito, Critical strong Feller regularity for Markov solutions to the Navier-Stokes equations, J. Math. Anal. Appl. 384 (2011), no. 1, 115-129.

96. M. Romito, The Martingale problem for Markov solutions to the Navier-Stokes equations, Seminar on Stochastic Analysis, Random Fields and Applications VI, Progr. Probab., vol. 63, Birkhäuser/Springer Basel AG, Basel, Basel, 2011, pp. 227-244.

97. M. Romito, Uniqueness and blow-up for a stochastic viscous dyadic model, Probab. Theory Related Fields 158 (2014), no. 3-4, 895-924.

98. M. Romito, L. XU, Ergodicity of the 3D stochastic Navier-Stokes equations driven by mildly degenerate noise, Stochastic Process. Appl. 121 (2011), no. 4, 673-700.

99. T. RUnSt, W. SiCKEL, Sobolev Spaces of Fractional Order, Nemytskij Operators, and Nonlinear Partial Differential Equations. Walter de Gruyter, 1996.

100. V. SCHEFFER, Turbulence and Hausdorff dimension, (Proc. Conf., Univ. Paris-Sud, Orsay, 1975), pp. 174-183. Lecture Notes in Math. 565, Springer, Berlin, 1976.

101. R. Schilling, L. PARtzsch, Brownian motion. An introduction to stochastic processes. De Gruyter Textbook. Berlin: de Gruyter 408 p. (2014).

102. H.-J. SChMEISSER, H. TRIEBEL, Topics in Fourier analysis and function spaces, Wiley-Interscience Publication, Chichester, 1987.

103. J. SERRIN, On the interior regularity of weak solutions of the Navier-Stokes equations, Arch. Rational Mech. Anal. 9 (1962), 187-195.

104. M. Siegert, M. Plischke, Solid-on-solid models of molecular-beam epitaxy, Phys. Rev. E, 50 (1994), 917-931.

105. B. Simon, The $P(\phi)_{2}$ Euclidean (quantum) field theory, Princeton University Press, Princeton, N.J., 1974, Princeton Series in Physics.

106. W. Stannat, Stochastic partial differential equations: Kolmogorov operators and invariant measures. Jahresbericht der Deutschen Mathematiker-Vereinigung, 113 (2011), no. 2, 81-109.

107. E. M. STEIN, Harmonic analysis: real-variable methods, orthogonality, and oscillatory integrals, Princeton Mathematical Series 43, Princeton University Press, Princeton, NJ, 1993.

108. O. Stein, M. WinkLer, Amorphous molecular beam epitaxy: global solutions and absorbing sets, European J. Appl. Math. 16 (2005), no. 6, 767-798.

109. D. W. Stroock, S. R. S. Varadhan, Multidimensional diffusion processes, Springer, Berlin, 1979.

110. T. Sun, H. Guo, M. GRAnt, Dynamics of driven interfaces with a conservation law, Phys. Rev. A 40 (1989), R6763-R6766.

111. H. S. G. SwANn, The convergence with vanishing viscosity of nonstationary Navier-Stokes flow to ideal flow in $R_{3}$, Trans. Amer. Math. Soc. 157 (1971), 373-397.

112. T. TAO, Global regularity for a logarithmically supercritical hyperdissipative Navier-Stokes equation, Anal. PDE 2 (2009), no. 3, 361-366.

113. T. TAO, Finite time blowup for an averaged three-dimensional Navier-Stokes equation, Preprint, (2014), arXiv:1402.0290v2 [math.AP], to appear in J. Amer. Math. Soc.

114. R. Temam, Navier-Stokes equations. Theory and numerical analysis, Studies in Mathematics and its Applications, 2. North-Holland Publishing Co., Amsterdam, 1984.

115. R. TEMAM, Infinite-dimensional dynamical systems in mechanics and physics, Applied Mathematical Sciences, 68. Springer-Verlag, New York, 1988.

116. JAN VAN NEERVEN, Stochastic Evolution Equations. ISEM Lecture Notes 2007/08.

117. J. Villain, Continuum models of crystal growth from atomic beams with and without desorption, J. Phys. I France, 1 (1991), 19-42.

118. J. B. WALSH, An introduction to stochastic partial differential equations, In École d'été de probabilités de Saint-Flour, XIV-1984, Lecture Notes in Math. 1180, pages 265-439. Springer, Berlin, 1986.

119. C. WANG, Well-posedness for the heat flow of biharmonic maps with rough initial data, Journal of Geometric Analysis, 22 (2012), no. 1, 223-243.

120. M. WINKLER, Global solutions in higher dimensions to a fourth-order parabolic equation modeling epitaxial thin-film growth, Z. Angew. Math. Phys. 62 (2011), no. 4, 575-608.

121. R. ZHU, X. ZHU, Three-dimensional Navier-Stokes equations driven by space-time white noise, Preprint, (2014), to appear in Journal of Differential Equations. 